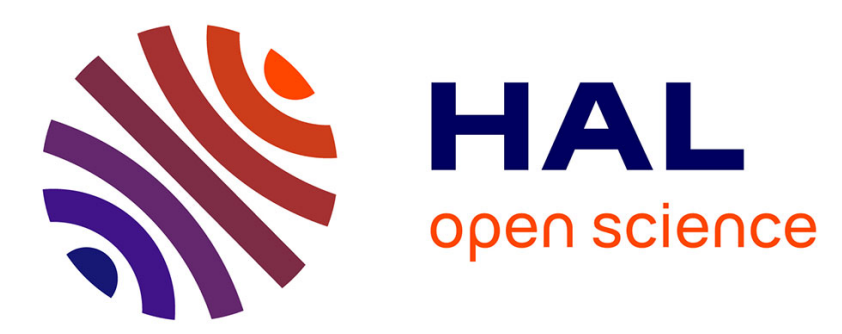

\title{
Hencky-type discrete model for pantographic structures: numerical comparison with second gradient continuum models
}

\author{
Emilio Turco, Francesco Dell'Isola, Antonio Cazzani, Nicola Luigi Rizzi
}

\section{- To cite this version:}

Emilio Turco, Francesco Dell'Isola, Antonio Cazzani, Nicola Luigi Rizzi. Hencky-type discrete model for pantographic structures: numerical comparison with second gradient continuum models. Zeitschrift für Angewandte Mathematik und Physik, 2016, 67 (4), pp.85. 10.1007/s00033-016-0681-8 . hal01345864

\author{
HAL Id: hal-01345864 \\ https://hal.science/hal-01345864
}

Submitted on 16 Jul 2016

HAL is a multi-disciplinary open access archive for the deposit and dissemination of scientific research documents, whether they are published or not. The documents may come from teaching and research institutions in France or abroad, or from public or private research centers.
L'archive ouverte pluridisciplinaire HAL, est destinée au dépôt et à la diffusion de documents scientifiques de niveau recherche, publiés ou non, émanant des établissements d'enseignement et de recherche français ou étrangers, des laboratoires publics ou privés. 


\title{
Hencky-type discrete model for pantographic structures: numerical comparison with second gradient continuum models
}

\author{
Emilio Turco, Francesco dell'Isola, Antonio Cazzani and Nicola Luigi Rizzi
}

\begin{abstract}
Hencky (Über die angenäherte Lösung von Stabilitätsproblemen im Raum mittels der elastischen Gelenkkette. Ph.D. thesis, Engelmann, 1921) proposed a discrete model for elasticae by introducing rigid bars and rotational springs. Hencky (Proc R Soc Lond A Math Phys Eng Sci 472(2185), 2016) approach has been introduced to heuristically motivate the need of second gradient continua. Here, we present a novel numerical code implementing directly the discrete Henckytype model which is robust enough to solve the problem of the determination of equilibrium configurations in the large deformation and displacement regimes. We apply this model to study some potentially applicable problems, and we compare its performances with those of the second gradient continuum model. The numerical evidence presented supports the conjecture that Hencky-type converges to second gradient model.
\end{abstract}

Keywords. Pantographic structures, Micro-mechanical model, Second gradient continuum, Nonlinear problems.

\section{Introduction}

In this paper, we introduce ab initio a discrete model for planar pantographic structures and we correspondingly formulate, using MATLAB, an effective computation tool which overcomes some technical difficulties confronted in $[3,4]$ via heavier finite elements-based computational schemes, whose formulation starts from second gradient continuum models. The developed computation tool, in particular, is better suited for determining the deformed shapes of pantographic sheets when: (1) large extensions are activated locally, (2) shear angles approach the value $\underset{2}{2}$ and (3) locking or "hourglass deformation effects" could arise due to the high heterogeneity of the material properties.

To explicit its effectiveness, we compare the newly developed computational tool with the one previously adopted using the FEM implemented in COMSOL Multiphysics (see [2]) based on a second gradient continuum model. The presented comparison considers some typical deformation problems including: (1) standard extension bias test; (2) shear/extension tests; (3) bending/extension tests; and (4) deformations induced by displacements imposed to single fibers (extraction tests).

The discrete model which we consider here includes both pairwise and triple elastic interactions between material particles and generalizes Hencky discrete model for the elastica [2,5-9].

The mechanical systems which could be described by the introduced discrete model include those considered and studied in $[2,4,5,10,11]$. Indeed, (1) the pivots interconnecting (see Fig. 1 for a 3D printing) the two arrays of beams are to be identified with the material particles which we introduce here; $(2)$ the fact that these pivots may store elastic energy may be efficiently modeled introducing suitable "shearing" springs (that is the rotational springs called $r_{3}$ in the following Figs. 3, 4); (3) the bending phenomena occurring between the two closest pivots and the corresponding stored elastic energy are modeled by the rotational springs called $r_{1}$ and $r_{2}$ in the following Figs. 3 and 4 ; and (4) the extensional phenomena occurring between two closer pivots are modeled by the extensional springs called $r_{0}$. 
However, we believe that the potential scope of the presented model is wider: it very likely may include the most relevant deformation phenomena in the woven fabrics described in [12-19]. Indeed, the fibers constituting these fabrics intersect one with the others very similarly than in pantographic structures, and in their deformation, one can still distinguish extension, bending and shear: even if the hypothesis of elastic behavior in woven fabrics is applicable in a smaller range of deformation states, the model presented here seems capable of catching some relevant aspects of its phenomenology. Similarly, some biological living tissues which are characterized by fibers embedded in a soft matrix can be described with the proposed model (see e.g., [20-24]).

In conclusion, we are sure that the synthetic discrete description given in this paper may supply some insight in all those phenomena where chains of forces may be activated by directional strain fields. A careful comparison with available experimental evidence will be the subject of some following papers.

After the concise statement concerning the original results, we complete this introduction by framing the presented results in the more general context of the mechanics of metamaterials and their continuum modeling. The dichotomy between discrete and continuum modeling will be shortly treated in a digression preceding the synthetic description of the structure of the paper.

\subsection{Metamaterials and their modeling with generalized continua}

The reader will find an increasing literature dealing with the class of materials which has been collectively gathered under the unifying concept of metamaterials. In this context, we have found particularly interesting the works by $[25,26]$, where metamaterials are described as those materials which, while not existing in Nature, are conceived by means of a theoretical abstraction and then engineered to have "exotic" properties.

Metamaterials are obtained by the "architectured" assemblage of multiple individual "microscopic" elements constituted by standard materials, usually arranged in (quasi-)periodic substructures [27]. It results that the macroscopic physical properties of metamaterials depend in a critical way on the topology of their microstructures and the nature of the interaction among constituting elements, while they are less sensitive to the standard properties of the micro-material constituting their microstructural elements. The macroscopic physical properties tailored in the design of metamaterial may be, for instance, optical, electromagnetic, thermal, mechanical and any combination (and coupling) of many of them. For instance, the particular shape, geometry, size, orientation and arrangement of the microstructural elements affect, in some particular metamaterials, the propagation of waves in a not-often-observed manner, e.g., not allowing the propagation in some band gaps (see, e.g., [28-31]) even if such a property is not present in the material constituting the microstructural elements (see also [32-35] for others unusual wave propagation phenomena).

1.1.1. Mechanical metamaterials. In the literature, more recently, a particular class of metamaterials has attracted the attention of mechanicians: the so-called mechanical metamaterials. The properties for which these architectured materials show unexpected or exotic features are purely mechanical. The newly synthesized metamaterial studied in [5] and considered here belongs to this class.

To give a hint of the possible performance of newly designed metamaterials, we list here some whose applications seem closer to those which we have in mind (for a more detailed discussion the reader is referred to $[3,36,37]):(1)$ it has been synthesized a (micro-)composite medium showing a negative effective bulk modulus, or a negative effective mass density, or both properties; (2) it is possible to design and fabricate metamaterials with negative Poisson's ratio; (3) it is possible to tailor materials showing selective damping effects using special material microstructures (see e.g., [38,39]).

The main problem in the theory of metamaterials is actually an inverse problem: given a continuum/discrete model (i.e., a set of kinematical parameters, either finite or infinite dimensional, and a 
Lagrangian action functional), one has to find if there actually exist mechanical systems which, at a specific length scale, behave as it is prescribed by the chosen (continuum or discrete) model.

The problem actually consists in finding the microscopic properties and structure of such mechanical systems to be able to construct (or how sometimes it is said with a word derived from Greek: synthesize) them, after having solved the corresponding technological problems.

The metamaterial conceived in $[2,5,9,40]$ has been designed to be governed, when considering macroscopic behavior, as a second gradient continuum and to have an enhanced extensional toughness: it has been actually synthesized exploiting the novel possibilities given by $3 \mathrm{D}$ printing technologies. This new class of bidimensional metamaterials, which have been also called extensible pantographic sheets, has been modeled using bidimensional continua which generalize those introduced in [41-43]: indeed they are conceived to account for the elastic energy in the extensional deformation and geodesic bending of constituting fibers simultaneously.

1.1.2. Generalized continuum models. Continuum mechanics is a powerful mean for the design of mechanical structures: indeed starting from the first half of nineteenth century this design has been based on the application of predictive mathematical models. Starting from the pioneering works by Navier and Piola (see the discussion in the following subsection), it was established that continuum models could be deduced from discrete microscopic ones: the underlying idea is that materials can be ultimately modeled at a micro-level as finite-dimensional Lagrangian systems and that their effective properties can be all obtained via a suitable homogenization procedure. However, the working assumptions put forward by Cauchy were later assumed to be of universal validity. The works of Piola on generalized continua (see [44]) and what has been later rediscovered and called peridynamics (see [45]) remained for a long time nearly unknown. The limiting assumptions accepted by Cauchy include the absence of length scale phenomena and of high contrast in microscopic properties. On the other hand, the particular class of microstructured mechanical systems which present high contrast $^{1}$ in microscopic properties once homogenized has been shown to produce generalized continua in [46-53].

Actually, the continuum model introduced by Cauchy is very accurate for a large class of phenomena but cannot be applied to all materials in every physical condition (and the contrary would be really surprising). When introducing generalized continua, the true conceptual frame settled by Cauchy and Navier is to be drastically modified. The concept of stress becomes secondary and the main role is played by deformation measures together with action and dissipation functionals (see [54] or e.g., [55-58]). The Euler-Lagrange equation obtained in this more encompassing modeling process cannot be anymore regarded to coincide with the balance of force unless one generalizes the concept of force. This can be done by introducing generalized actions as the dual quantities in the work of the gradients of displacements (see e.g., [59-62] or [63-65] for 2D or 1D generalized continua characterized by a specific microstructure).

All these difficulties are easily surmounted when discrete models are usable: what we prove is possible in the present paper. Moreover, a further consideration is needed here: at the end a continuum model, for getting predictions, needs to be discretized. In other words, in any case a discrete model is used, whose structure is rather out of the control of the modeler as it is mediated by many complex steps. In the case of pantographic structures, they can be shortly listed as follows: (1) to find the right class of generalized continua which is able to capture their behavior (and it seems that such class must be at least the class of second gradient Piola continua, if not the class of micro-stretch continua); (2) to find the correct FEM for getting algorithms converging to the physically meaningful shapes of introduced continua; and (3) to establish the correct identification of constitutive parameters for getting physically founded predictions.

Here, we control totally the modeling process by introducing a convincing discrete model, we formulate a Lagrangian equilibrium condition, and we find with a robust code under our complete control, since

1 These systems are defined as quasiperiodical systems having some of the physical properties diverging when the size of the representative elementary volume tends to zero, while simultaneously some others properties are vanishing in this limit. Pantographic structures verify this definition, see [2]. 
it has been developed by ourselves, to determine all equilibrium configurations. The comparison with the previously obtained results found in $[3,5]$ is really comforting: there is a total correspondence of the predictions. When one of the two models fails to converge, it is always the one obtained via the mediation of generalized continuum theories.

\subsection{A historical perspective}

The controversy about the prevalence of continuum models on discrete models (or vice versa, depending on the tastes and the schools) for mechanical (or more generally physical) systems is very old. Probably, it started with the antithesis between Epicurean atomism and Heraclitean continuum approach: the more recent dispute between Mach and Boltzmann is an example of how bitter this contraposition may become. In this paper, we do not try a historical analysis of this contraposition and we do not dare to take position on the aforementioned controversy. Actually, we believe that one has to assume a pragmatic view on the question. In general, a mathematical model to be used for describing physical systems has to have the following obvious features: (1) it has to be formulated based on a clear minimal set of postulates which should be self-consistent and (2) it has to be predictive when applied to describe physical phenomena.

In order to get predictions out of a set of postulates, some exercises of mathematics need to be solved: the obtained solutions, when engineering applications are needed, must involve the performance of specific computations, which allow for the exact quantitative verification of considered models and for the precise design of novel devices.

Before the advent of modern computing machines, the most powerful computing tool has been given by mathematical analysis and its ancillary disciplines, i.e., the theories of analytical, elementary or special functions. In fact, once a model was postulated on the basis of a variational principle (see, e.g., $[44,45,66])$ then the corresponding Euler-Lagrange conditions were found in order to be able to apply for their solutions all the methods which are made available by mathematical analysis. We refer, for instance, to the approximate methods of solutions by series, by polynomial approximations, separation of variables, or to the so-called analytical or semi-analytical methods. Instead discrete mathematics, and in particular the theory of discrete difference equations, could not produce calculation methods based on closed form solutions, as it is done, instead, very effectively by infinitesimal analysis. As a consequence, also those scientists who, being followers of Epicurean school, believed in the atomistic and discrete fundamental nature of matter did study continuum models. Piola [66], Navier [67], and later Boltzmann [68] (just to name a few of them) started their postulation starting with a discrete finite-dimensional model where Lagrangian coordinates consist of $N$-tuples of material particles coordinates and then, via suitable homogenization methods, deduced the governing equations of the continuum approximating one.

Therefore, without the modern powerful tools of automatic computation, also the Epicureans needed to deduce continuum models to get applicable equations from which, via suitable analytical and simple numerical computations, they could get quantitative predictions.

A paradoxical situation was then created. Continuum models, which had been first created as auxiliary tools needed for performing calculations and obtained via a deductive process, were regarded subsequently as constituting the most fundamental ones, simply because they were considered the most effective way for describing macroscopic phenomena (see [69]). The basic postulates in these continuum models have been identified with a list of balance laws (see [44]) to which a (presumed) firmer physical meaning has been (more or less arbitrarily) attributed. Subsequently, discrete models were introduced as an ancillary tool for getting effective calculation algorithms: variational principles for continuum models were therefore deduced as "theorems" (and it did not seem to many an oxymoron to talk about the theorem (sic!) of the principle of virtual work) to be used for introducing the continuum model discretization. Actually, as the historical circumstances were changed, discrete models had become the most suitable ones for supplying quantitative predictions, as von Neumann machines are able to calculate the solution of algebraic equations having millions of unknowns. 
This shift on the role given, in the passage from a cultural paradigm (in the sense of Kuhn [70]) to another paradigm, to the same concept is not new in the history of science. One concept may be considered as "fundamental" by a school and "ancillary" in another one and vice versa. We postpone a careful study of these cultural phenomena to further investigations as it seems that it plays a major role also in mechanical sciences. Simply we want to recall here (see, e.g., [71]) that in Hellenistic science a predictive model describing the motion of planets seems to have been formulated. The main tool for calculating these motions was some mechanisms (like Antikythera Machine see [72]) whose functioning mechanism was epicyclic gears: Ptolemy, being a practical astronomer, instead of teaching the underlying astronomic theory decided to describe simply the theory needed for constructing the relative calculation ancillary tools. As a consequence, during Middle Age, the astronomers believed to the existence of several heavens around the Earth, the closer ones embedding the "moving planets" which were supported by moving structures and the most distant one being constituted by a fixed vault on which also fixed stars were blocked. The cultural shift was again of the same nature: those concepts, which had been conceived as secondary ones needed for supplying calculating tools, did eventually become the most fundamental ones, assumed as the basis of the whole theoretical speculation.

\subsection{Structure of the paper}

In Sect. 2, the Lagrangian finite-dimensional model proposed and studied in this paper is introduced. The spirit of Piola, when he describes the molecular mechanical system which he places at the foundation of his presentation, is faithfully followed. A lattice of positions is defined which characterizes the reference configuration of all particles constituting the system. Lagrangian mechanics formalism is then assumed: a deformation energy is associated with the set of interaction springs introduced and the problem of its minimization is presented.

In Sect. 3, a solution strategy of the aforementioned minimization problem is presented, and all the details needed for its eventual reproduction are given.

In Sect. 4, some numerical solutions are shown for some equilibrium problems related to engineering applications. In particular there are presented solutions which are relevant to standard extension bias test with the correspondent reactive force distributions and resultants induced by imposed displacements on constraints.

The reader will remark that in the dedicated literature the determination of reactive forces for bias test is considered somehow difficult. Moreover, some equilibrium shapes are shown in the presence of displacements imposed on single fibers and in the test of extraction of a fiber.

In Sect. 5, the performance of the presented numerical model is compared with those shown in [2]. The code which is presented here needed an ad hoc programming activity while previously the code was obtained by using a standard FE integrator (COMSOL). As expected, the convergence capacities (to solution having a correct physical interpretation) of the newly presented model are improved remarkably. Of course, with FE codes one can improve the results related to higher gradient continuum theories using newly developed numerical tools with an intrinsic high continuity as done, e.g., in [73,74,74-80]; however, herein we want to explore the potential of the proposed discrete model whose features are based on the microstructure properties of the system under study and compare its performances with already tested and verified models.

In conclusions, the future immediate research perspectives are listed and some major possible modeling improvements are delineated.

\section{Hencky-Piola-type model for planar pantographic sheets}

As discussed in Introduction, among the many modeling possibilities, we have chosen, in this paper, to introduce a finite-dimensional Lagrangian model for describing pantographic planar sheets. 


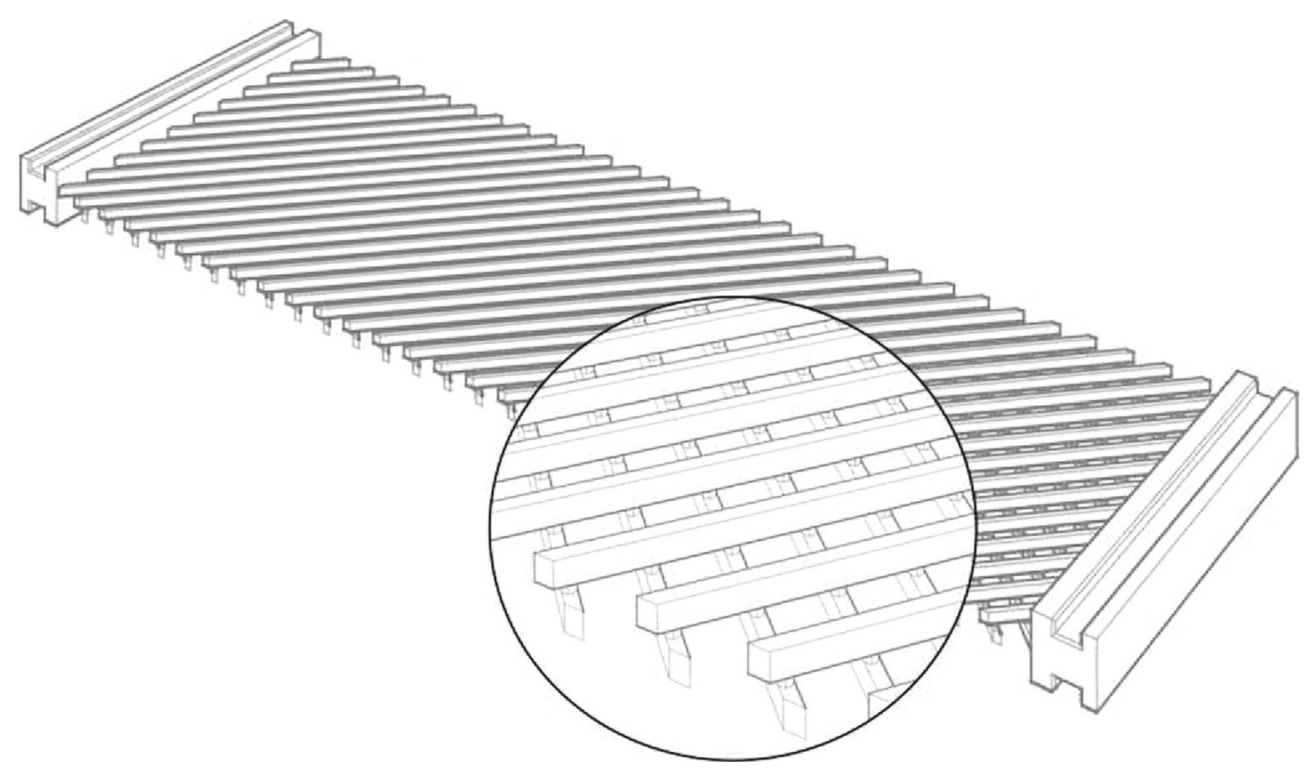

FIG. 1. Pantographic structure

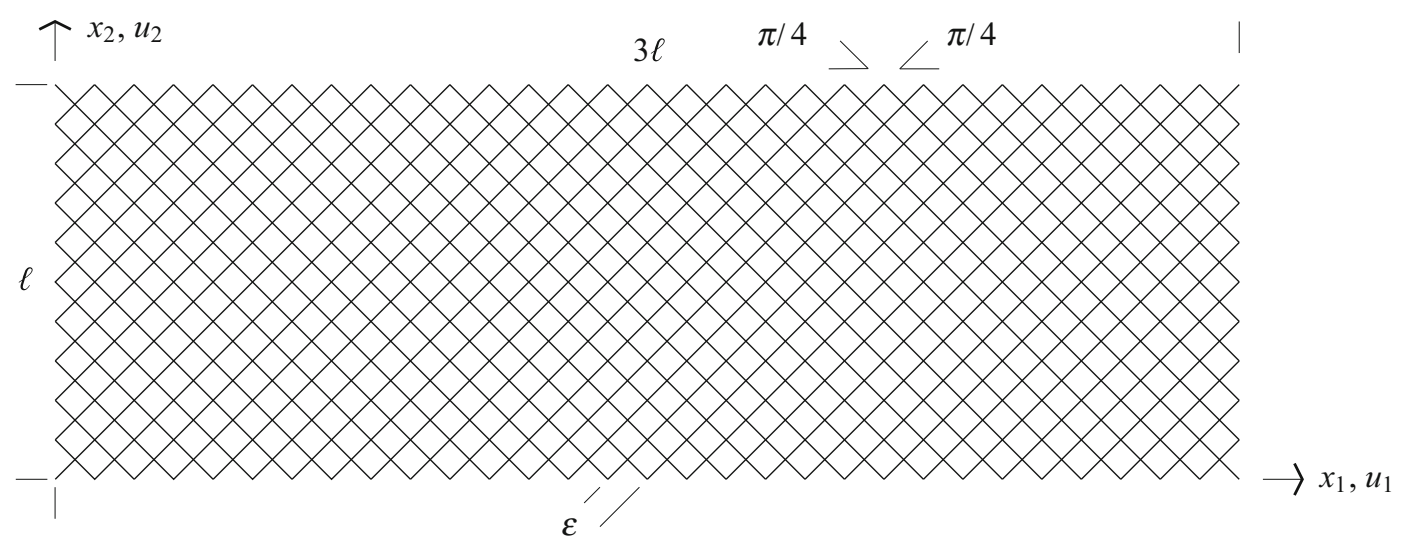

FIG. 2. Pantographic lattice

This model is Piola type as we consider a finite number of material particles occupying, in a reference configuration, the nodes of a rectangular square cell lattice having length $\varepsilon$ (see Fig. 2, where it was assumed that $n_{f}=10$ square cells constitute the shorter side of the rectangular lattice).

The lattice is, roughly speaking, composed by two arrays of fibers which are oriented at an angle $\pi / 4$ and $-\pi / 4$ with respect to $x_{1}$ axis: we will call array 1 that at the angle $\pi / 4$ and array 2 the other one.

Each of the introduced material particles models the pivots which in the 3D printed specimen interconnect the two considered arrays of beams, see Fig. 1.

This model is Lagrangian because we introduce the reference position of the generic $i$ th material particle via the position $P_{i}$ and we consider as a Lagrangian kinematical parameter for each of them the actual position, denoted by $p_{i}$. 


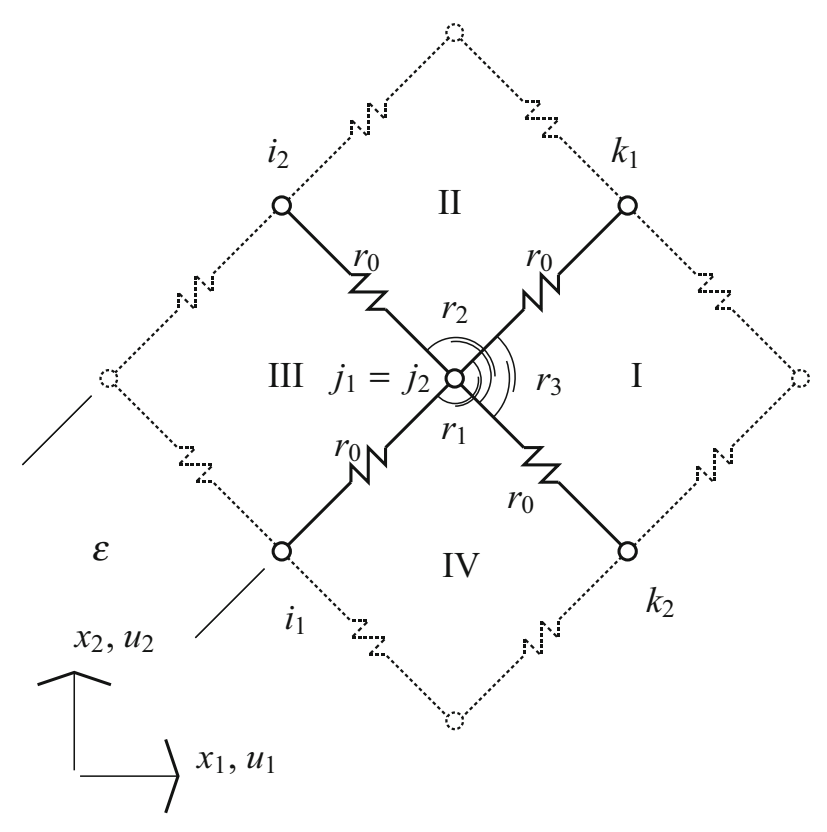

FIG. 3. Discrete mechanical model

This model is also a Hencky type one because, as done in his pioneering work (see $[1,6-8]$ ) for elasticae, we model the elastic interactions among the particles by means of rotational and extensional springs such as those represented in Fig. 3, so that we assume to need pairwise and triple particles interactions.

Our conjecture is that it is formulated, in this way, a discrete mathematical model which is able to supply an accurate prediction of the structural response in the regime of (very) large displacements.

The modeling procedure is to be completed by defining the deformation energy of each spring element. The problem of determining equilibrium configurations is successively solved by imposing the stationarity of total potential energy following the algorithm described in Sect. 3.

Our Ansatz concerning deformation energies can be summarized as follows:

Deformation energy for axial springs Each axial spring stores elastically an energy which depends quadratically on its variation of length:

$$
w_{0}=\frac{1}{2} r_{0}\left(\left\|p_{j}-p_{i}\right\|-\varepsilon\right)^{2}
$$

where $p_{i}$ and $p_{j}$ are the actual position, see Figs. 3 and 4 , of the nodes connected by the considered extensional spring whose rigidity is denoted $r_{0}$ while $\varepsilon$ is the initial unstressed length of the axial spring.

Deformation energy for bending springs Three consecutive particles along array 1 or 2 interact via a rotational spring whose stored energy depends on the angle formed by the two consecutive segments connecting, in the actual configurations, the particles' positions:

$$
w_{1,2}=r_{1,2}\left(\cos \gamma_{1,2}+1\right),
$$

where

$$
\cos \gamma_{1,2}=\frac{\left\|p_{j_{1,2}}-p_{i_{1,2}}\right\|^{2}+\left\|p_{k_{1,2}}-p_{j_{1,2}}\right\|^{2}-\left\|p_{k_{1,2}}-p_{i_{1,2}}\right\|^{2}}{2\left\|p_{j_{1,2}}-p_{i_{1,2}}\right\|\left\|p_{k_{1,2}}-p_{j_{1,2}}\right\|},
$$

and $p_{i_{1,2}}, p_{j_{1,2}}$ and $p_{k_{1,2}}$ are the ordered actual position of the three aligned nodes along array 1 or 2 and $r_{1}$, see Figs. 3 and 4 , and $r_{2}$ denote the rigidities of the corresponding rotational springs. 

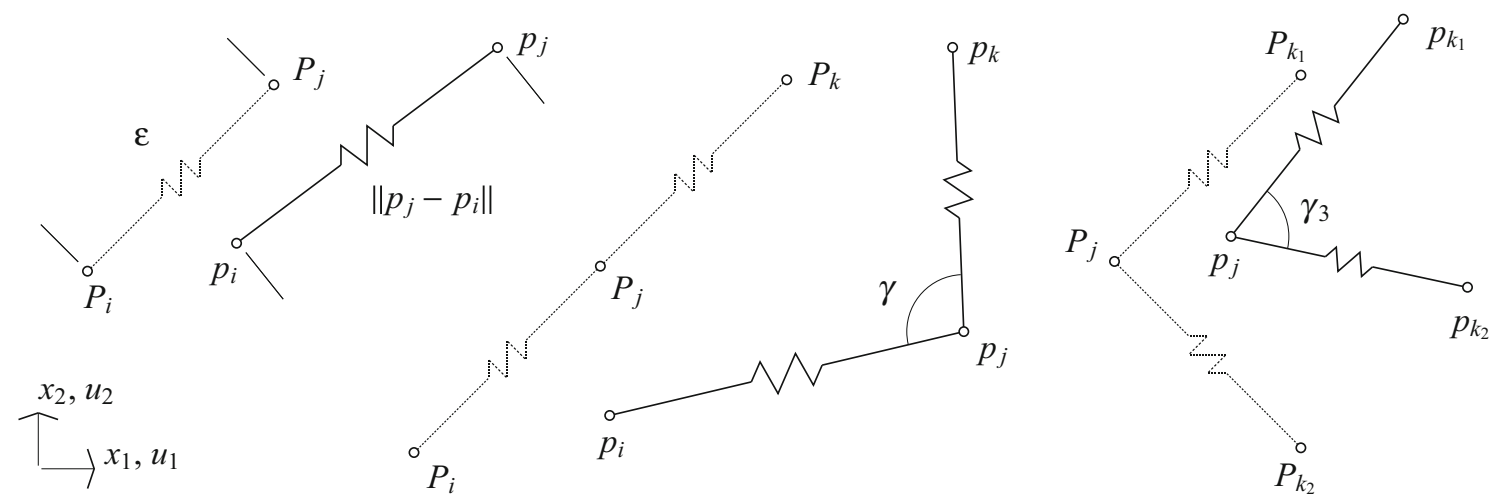

FIG. 4. Kinematics of extensional, bending and shear springs

The deformation energies for springs at the nodes where displacement is imposed need the specification of suitable boundary conditions. In particular, if the short sides of the lattice are constrained to be fixed on two rigid bodies and the bending springs are connected with material segments of these bodies then the previous formulae obviously supply the needed relations when setting (if the constrained node $j$ has not the subsequent node $k$ in the interior of the lattice)

$$
p_{k_{1,2}}=P_{k_{1,2}}+R_{k_{1,2}}
$$

or (if the constrained node $j$ has not the preceding node $i$ in the interior of the lattice)

$$
p_{i_{1,2}}=P_{i_{1,2}}+R_{i_{1,2}},
$$

where $R_{k_{1,2}}$ or $R_{i_{1,2}}$ represent the corresponded rigid displacement.

Deformation energy for shear springs Via the interconnecting pivots, the two arrays of fibers interact elastically: if the actual angle between the two arrays is $\gamma_{3}$ we assume that the stored energy depends quadratically on the difference $\left(\gamma_{3}-\frac{\pi}{2}\right)$

$$
w_{3}=\frac{1}{2} r_{3}\left(\gamma_{3}-\frac{\pi}{2}\right)^{2}
$$

where (see Figs. 3, 4)

$$
\cos \gamma_{3}=\frac{\left\|p_{j_{1}}-p_{k_{2}}\right\|^{2}+\left\|p_{k_{1}}-p_{j_{1}}\right\|^{2}-\left\|p_{k_{1}}-p_{k_{2}}\right\|^{2}}{2\left\|p_{j_{1}}-p_{k_{2}}\right\|\left\|p_{k_{1}}-p_{j_{1}}\right\|},
$$

if $p_{j_{1}}$ and $p_{k_{1}}, p_{k_{2}}$ are the actual position of the nodes relative to the rotational spring of rigidity $r_{3}$ connecting array 1 with array 2 . Remark that we assume to have one of such shear springs also in quadrants $I I, I I I$ and $I V$, see Fig. 3 .

The strain energy defined for each kind of introduced springs supplies a Hencky-type model for the pantographic sheet and allows us to easily define the total strain energy by simply summing each contribution.

In order to have a complete solution of the considered equilibrium problem, displacements and forces or couples exerted by each spring, a step-by-step procedure was implemented to reconstruct the complete equilibrium path of the pantographic sheet, as it will be described in detail in the following Sect. 3. 


\section{The solution algorithm}

We collect the nodal displacements in the lattice in the vector $\mathbf{d}$ in order to write the total energy of the pantographic structure in the form:

$$
W(\mathbf{d})=\sum_{e} w_{0}+w_{1}+w_{2}+w_{3}-L_{\mathrm{ext}},
$$

where $e$ ranges on all the spring, extensional, bending and shear and $L_{\text {ext }}$ is the work of the external loads and all quantities on the $R H S$ depend on the variable $\mathbf{d}$.

The equilibrium problem which we want to consider is a mixed one: we assume that the displacements of some particles are imposed and that some external conservative forces are applied to the remaining particles. Let us therefore decompose $\mathbf{d}$ into the pair composed by two vectors: the imposed displacements $\mathbf{u}_{a}$ and the free displacements $\mathbf{u}$. For notational aims, we will reorder $\mathbf{d}$ to get the decomposition

$$
\mathbf{d}=\left(\mathbf{u}, \mathbf{u}_{a}\right) .
$$

Because of our assumption, we have that $L_{\text {ext }}$ depends only on $\mathbf{u}$.

The nonlinear system of equilibrium equations is obtained by imposing that the first variation of $W$ vanishes:

$$
\mathbf{s}(\mathbf{u})-\mathbf{p}(\mathbf{u})=\mathbf{0}
$$

where $\mathbf{p}(\mathbf{u})$ is the vector which collects the Lagrangian components of external forces (which may be assumed to be dead loads, for instance, so that $\mathbf{p}$ becomes independent of $\mathbf{u})$ and $\mathbf{s}(\mathbf{u})$ is the vector of the internal forces (called also, in the context of structural mechanics, structural reaction), as defined by:

$$
\mathbf{s}(\mathbf{u})=\frac{\mathrm{d} W}{\mathrm{~d} \mathbf{u}}, \quad \mathbf{p}(\mathbf{u})=\frac{\mathrm{d} L_{e}}{\mathrm{~d} \mathbf{u}} .
$$

The tangent stiffness matrix is defined as the derivative of the structural reaction $\mathbf{s}(\mathbf{u})$ with respect to the displacements vector $\mathbf{u}$, in formulae:

$$
\mathbf{K}_{T}(\mathbf{u})=\frac{\mathrm{d} \mathbf{s}(\mathbf{u})}{\mathrm{d} \mathbf{u}}=\frac{\mathrm{d}^{2} W}{\mathrm{~d} \mathbf{u}^{2}},
$$

If the external load potential depends on a parameter $\mu$ and the imposed displacements depend on a parameter $\lambda$ (as in the case of quasi-static loading and imposed displacements), then Eq. (7) becomes:

$$
\mathbf{r}(\mathbf{u}, \lambda, \mu):=\mathbf{s}\left(\mathbf{u}, \mathbf{u}_{a}(\lambda)\right)-\mathbf{p}(\mathbf{u}, \mu)=\mathbf{0}
$$

An equilibrium manifold is defined as a surface $\mathbf{u}(\lambda, \mu)$ such that

$$
\mathbf{r}(\mathbf{u}(\lambda, \mu), \lambda, \mu)=\mathbf{0} \text {. }
$$

For example, in the case of an external dead load, we will have that $\mathbf{p}$ is independent of $\mathbf{u}$, so that if a linear dependence on $\mu$ is assumed, we can set:

$$
\mathbf{p}(\mu)=\mathbf{p}_{0}+\mu \hat{\mathbf{p}},
$$

Moreover, when the equilibrium equation (7) can be linearized in the neighborhood of the solution $\mathbf{u}_{0}$ relative to the dead load $\mathbf{p}_{0}$ then, as $\mathbf{s}\left(\mathbf{u}_{0}\right)-\mathbf{p}_{0}=\mathbf{0}$ and setting $\left(\mathbf{u}-\mathbf{u}_{0}\right)=: \Delta \mathbf{u}$ we have

$$
\mathbf{0}=\mathbf{s}(\mathbf{u}(\mu))-\mathbf{p}_{0}-\mu \hat{\mathbf{p}} \simeq \mathbf{s}\left(\mathbf{u}_{0}\right)-\mathbf{p}_{0}+\mathbf{K}_{T}\left(\mathbf{u}_{0}\right) \Delta \mathbf{u}-\mu \hat{\mathbf{p}}=\mathbf{K}_{T}\left(\mathbf{u}_{0}\right) \Delta \mathbf{u}-\mu \hat{\mathbf{p}},
$$

from which we deduce

$$
\Delta \mathbf{u}=\mu\left(\mathbf{K}_{T}\left(\mathbf{u}_{0}\right)\right)^{-1} \hat{\mathbf{p}}
$$

which will be of use in the sequel.

The solution of the nonlinear equilibrium system of Eq. (10) can be found by means of an incremental iterative procedure based on the Newton-Raphson scheme. 
As we will limit ourselves to the case of equilibrium paths depending only on imposed displacements, we introduce here only the parameter $\lambda$. Starting from an estimated point of the equilibrium path $\left(\lambda_{j}, \mathbf{u}_{j}\right)$ verifying the condition

$$
\left\|\mathbf{r}\left(\mathbf{u}_{j}, \lambda_{j}\right)\right\| \leq \eta
$$

i.e., with a pair being an $\eta$-approximate solution of the equilibrium condition (7), the iterative scheme, once the step $\Delta \lambda$ is fixed, is obtained by constructing the $\eta$-approximate solution $\left(\mathbf{u}_{j+1}=\right.$ : $\left.\mathbf{u}_{j}+\Delta \mathbf{u}_{j}, \lambda_{j+1}:=\lambda_{j}+\Delta \lambda\right)$ by using a sub-iteration scheme for calculating $\Delta \mathbf{u}_{j}$.

This sub-iteration is specified by the following steps

1. We calculate the so-called residual nodal forces relative to the initial tentative value $\Delta \mathbf{u}_{j, 0}\left(\mathbf{u}_{j}, \lambda_{j}\right)=$ 0

$$
\mathbf{r}\left(\mathbf{u}_{j}, \lambda_{j}+\Delta \lambda\right)=: \mathbf{p}_{j, 0}
$$

2. Once calculated the $h$ th approximation $\sum_{l=0}^{h} \Delta \mathbf{u}_{j, l}\left(\mathbf{u}_{j}, \lambda_{j}\right)$ of the increment $\Delta \mathbf{u}_{j}$, we calculate the corresponding residual

$$
\mathbf{r}\left(\mathbf{u}_{j}+\sum_{l=0}^{h} \Delta \mathbf{u}_{j, l}\left(\mathbf{u}_{j}, \lambda_{j}\right), \lambda_{j}+\Delta \lambda\right)=: \mathbf{p}_{j, h} .
$$

3. If the inequality

$$
\left\|\mathbf{p}_{j, h}\right\|>\eta
$$

is verified we set, on the basis of (12) and assuming $\mu=-1$ (this is equivalent to trying to find the solution by "imposing" fictitious external dead loads being opposed to the calculated residuals)

$$
\Delta \mathbf{u}_{j, h+1}=-\left(\frac{\partial \mathbf{r}\left(\mathbf{u}_{j}+\sum_{l=0}^{h} \Delta \mathbf{u}_{j, l}\left(\mathbf{u}_{j}, \lambda_{j}\right), \lambda_{j}+\Delta \lambda\right)}{\partial \mathbf{u}}\right)^{-1} \mathbf{p}_{j, h}
$$

and continue the sub-iteration.

4. If the inequality

$$
\left\|\mathbf{p}_{j, h}\right\| \leq \eta
$$

is verified, then we set

$$
\Delta \mathbf{u}_{j}:=\sum_{l=0}^{h} \Delta \mathbf{u}_{j, l}\left(\mathbf{u}_{j}, \lambda_{j}\right)
$$

and we stop the iteration.

\section{Some numerical results}

The geometrical parameters considered to model the pantographic structure are:

$$
\begin{aligned}
\ell & =70 \mathrm{~mm}, \\
\varepsilon & =\frac{\sqrt{2}}{2} \frac{\ell}{n_{f}},
\end{aligned}
$$

where $n_{f}$ is the number of fibers which intercept the smaller side of the lattice, e.g., in Fig. $2 n_{f}=10$ and consequently $\varepsilon \approx 4.95 \mathrm{~mm}$. 


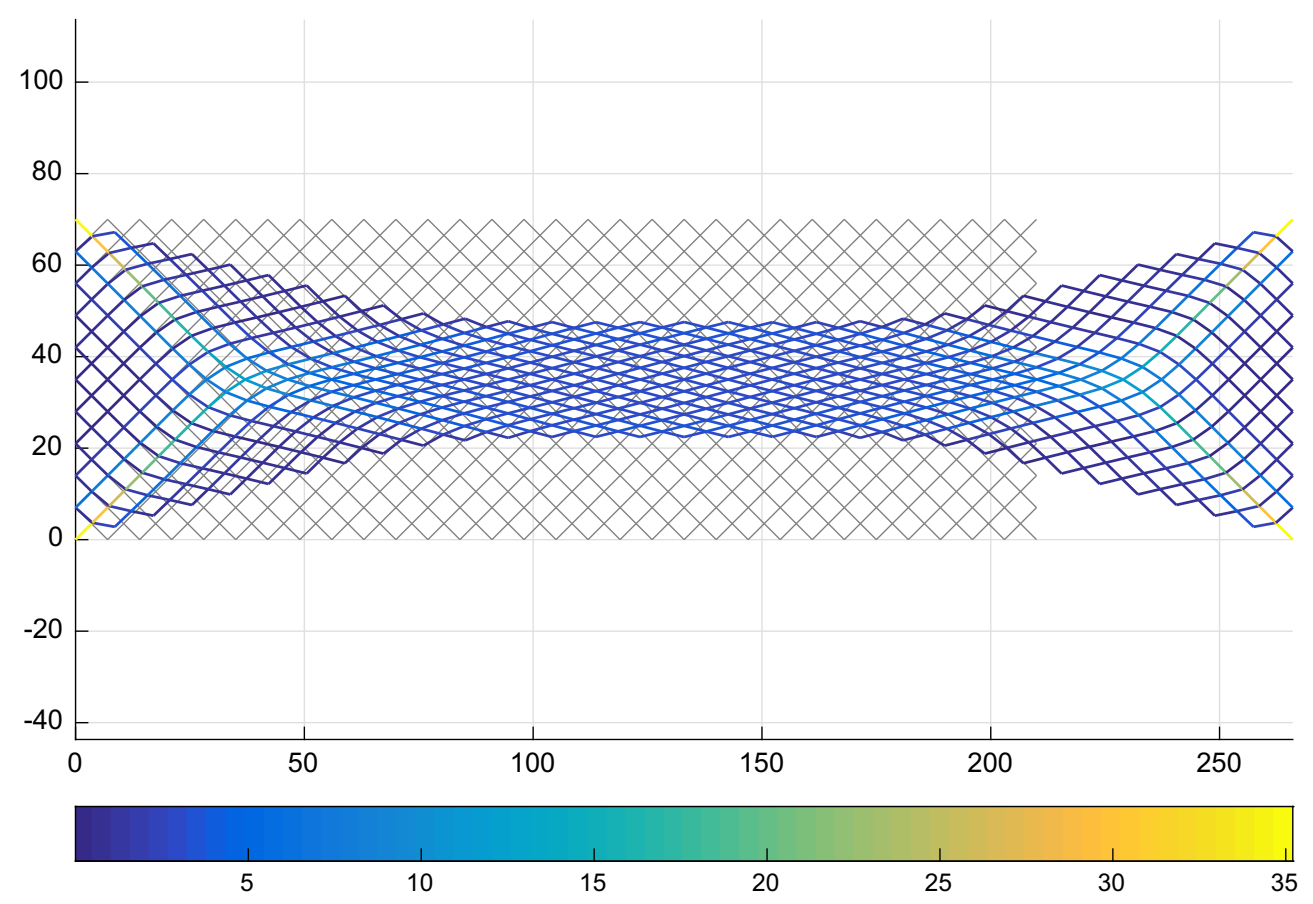

FIG. 5. Bias test for $n_{f}=10$ : reference configuration (gray), deformation and color bar of the internal forces on extensional springs (color figure online)

The mechanical parameters of the lattice are the rigidities of the springs for which the following values are used:

$$
\begin{aligned}
& r_{0}=134 \mathrm{Nmm}, \\
& r_{1}=r_{2}=19.2 \mathrm{~N} \mathrm{~mm}, \\
& r_{3}=\bar{k}_{3} \varepsilon^{2}=\frac{3.90}{4} \mathrm{Nmm} .
\end{aligned}
$$

\subsection{Standard extension bias test}

This test is characterized by assuming, see again Fig. 2:

$$
\begin{aligned}
u_{1}\left(0, x_{2}\right) & =u_{2}\left(0, x_{2}\right)=u_{2}\left(3 \ell, x_{2}\right)=0, \\
u_{1}\left(3 \ell, x_{2}\right) & =0.8 \ell=56 \mathrm{~mm} .
\end{aligned}
$$

In Fig. 5, the deformation of the lattice, compared with its reference position (in gray), along with the color bar which indicates the level of the internal forces on the extensional springs for the case $n_{f}=10$ is reported. For the same test, Fig. 6 a shows the global reaction of constraints on the side $x_{1}=3 \ell$, more precisely the $x_{1}$ component in blue and the $x_{2}$ component in red, as a function of the parameter $\lambda \in(0,1)$ which guides the imposed displacements from 0 to $u_{1}\left(3 \ell, x_{2}\right)$. Figure $6 \mathrm{~b}$ shows the discrete density $r\left(x_{2}\right)$ of the global reaction on $x_{1}=0$ when $\lambda=1$ (the $x_{1}$ component in blue and the $x_{2}$ component in red), and Fig. 6c shows the axial $E_{a}(\lambda)$, bending $E_{b}(\lambda)$ and shear $E_{s}(\lambda)$ energies. Figure 7 reports, always 


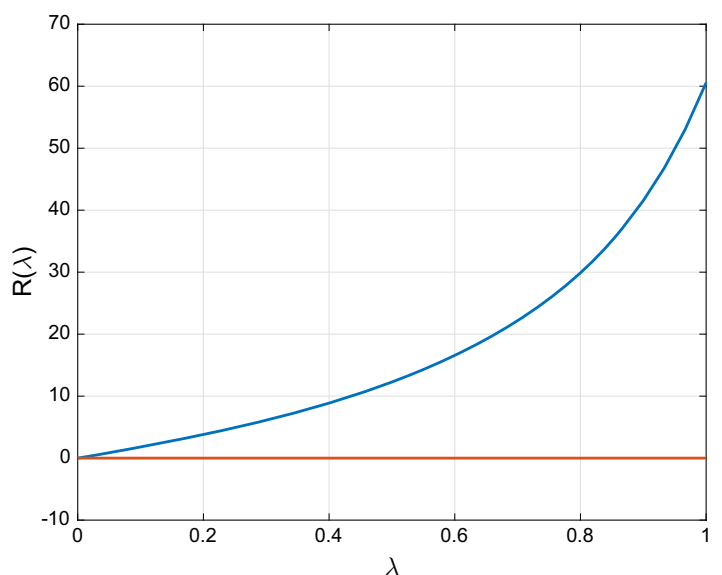

(a)

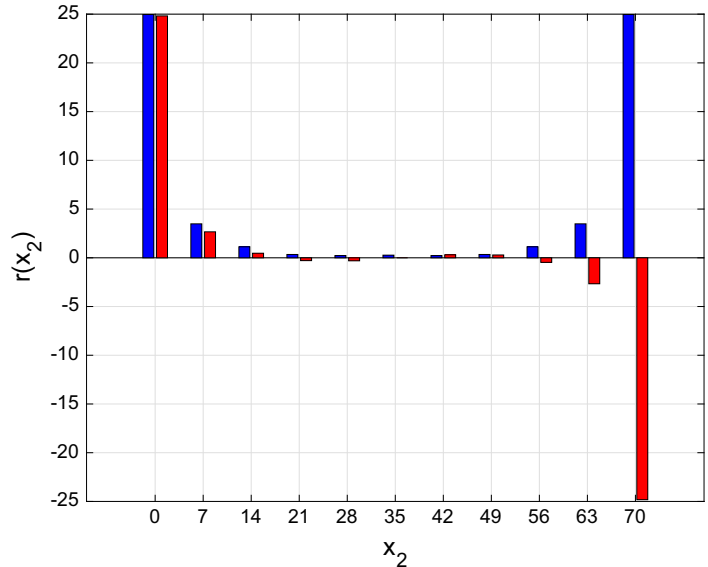

(b)

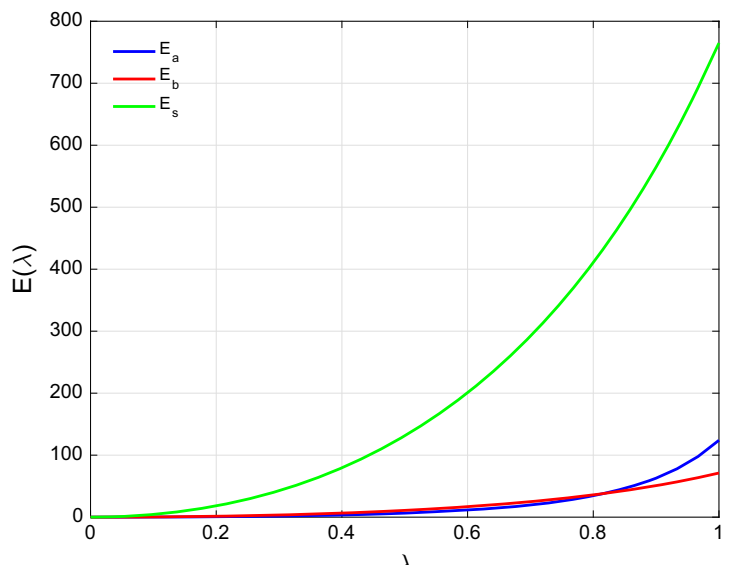

(c)

FIG. 6. Bias test for $n_{f}=10$ : global reaction of constraints $R(\lambda)(\mathbf{a})$, its density $r\left(x_{2}\right)$ when $\lambda=1$ (b) and strain energies (c). a $x_{1}$ component in blue and $x_{2}$ component in red. $\mathbf{b} x_{1}$ component in blue and $x_{2}$ component in red. $\mathbf{c}$ axial, $E_{a}$ (blue), bending, $E_{b}(r e d)$, and shear, $E_{s}$ (green), strain energy versus $\lambda$ (color figure online) 


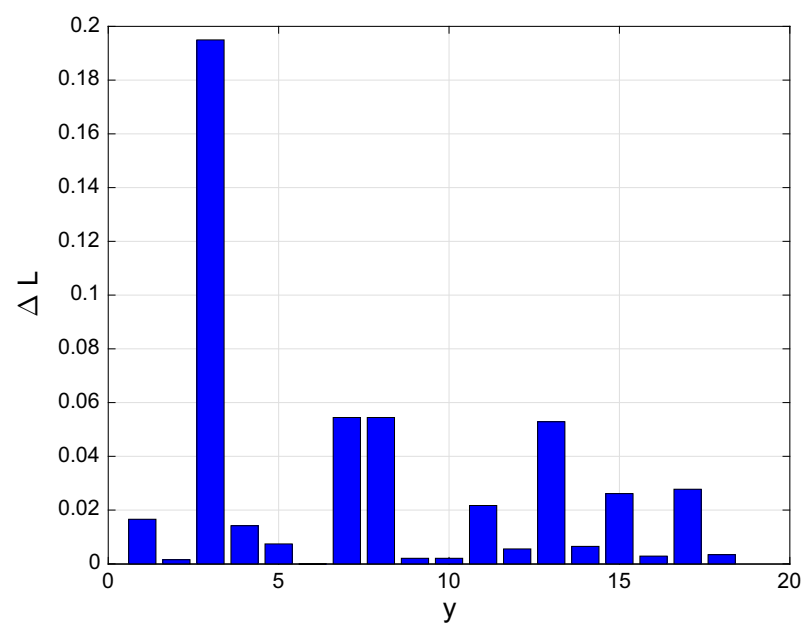

FIG. 7. Bias test for $n_{f}=10$ : spring elongation along $x_{2}=\sqrt{2} \varepsilon+x_{1}$

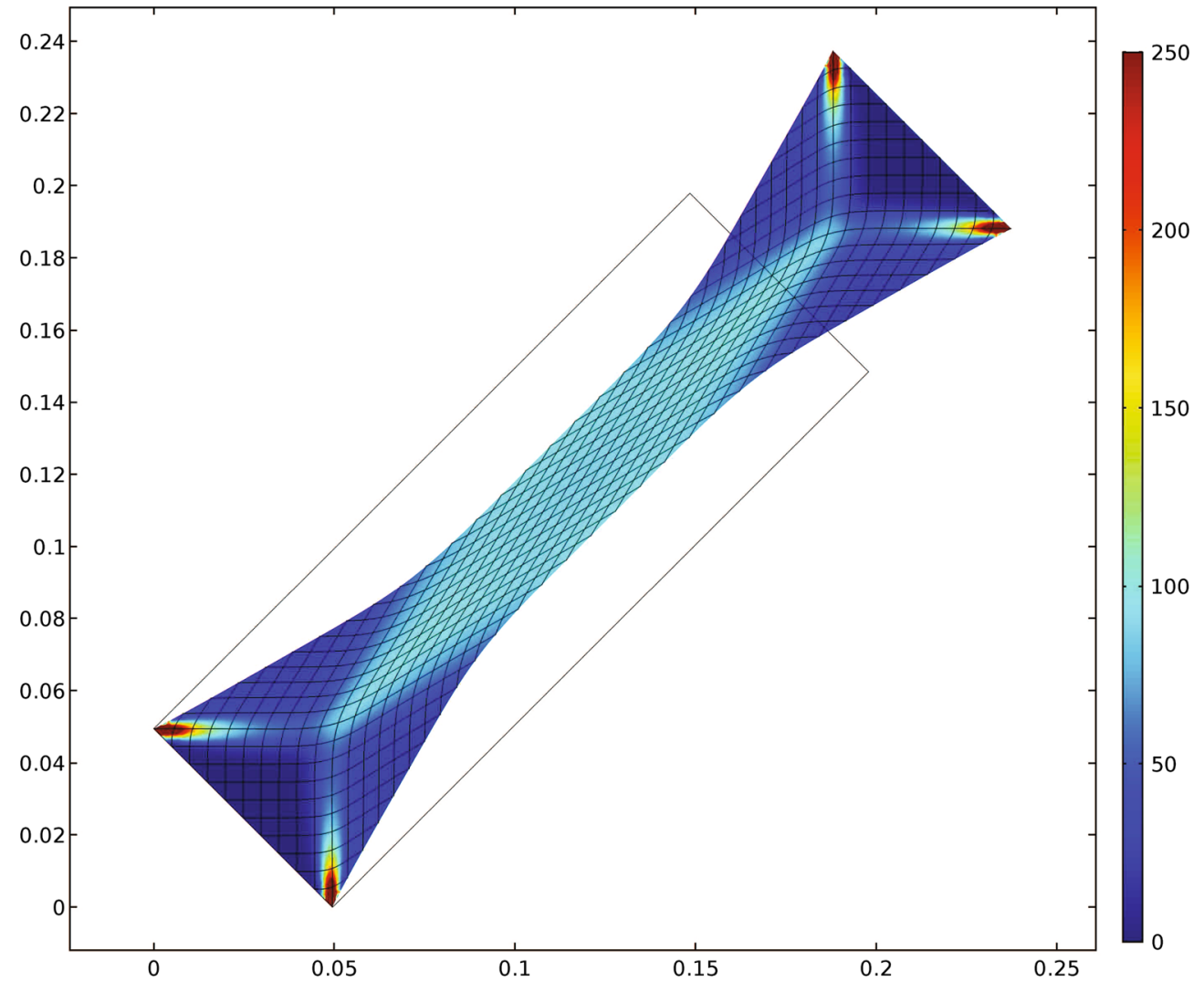

Fig. 8. Bias test performed by using a second gradient model: reference configuration (gray), deformation and color bar of the strain energy (color figure online) 


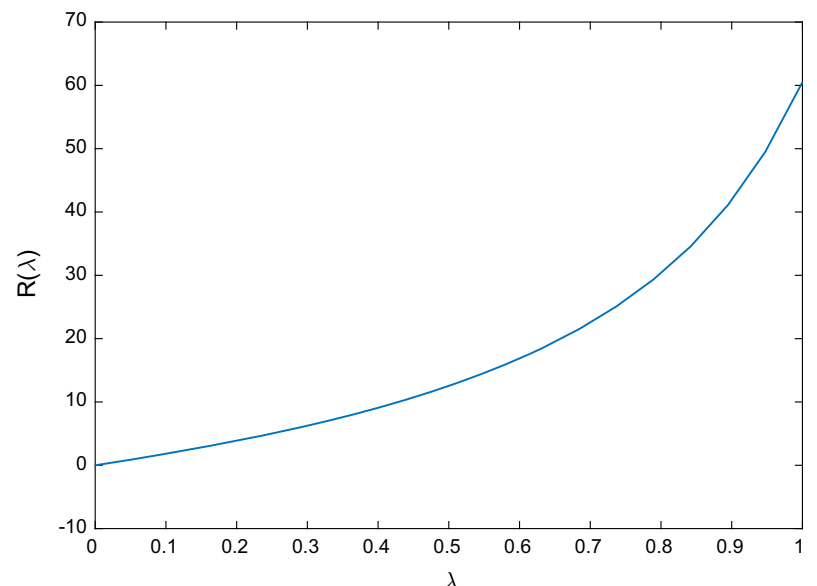

(a)

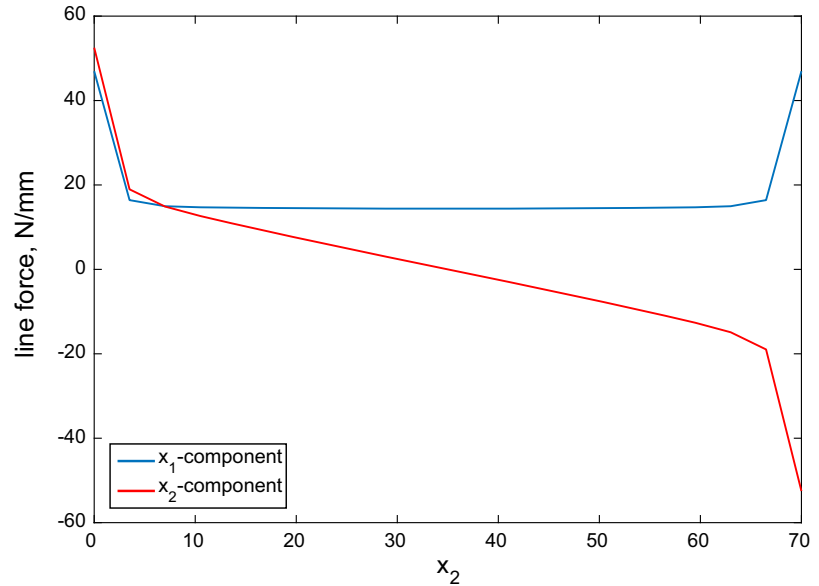

(b)

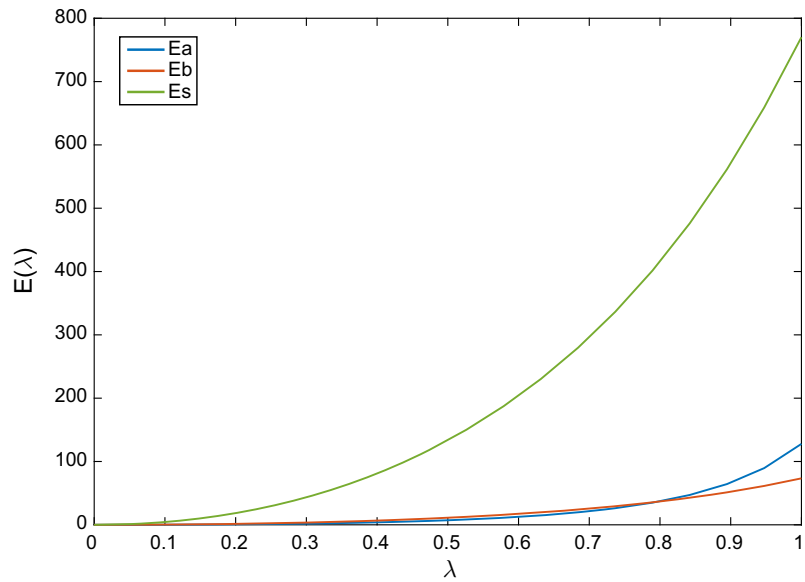

(c)

FIG. 9. Bias test performed by using a second gradient model: global reaction of constraints $R(\lambda)(\mathbf{a})$, its density $r\left(x_{2}\right)$ when $\lambda=1$ (b) and strain energies (c). a $x_{1}$ component in blue. b $x_{1}$ component in blue and $x_{2}$ component in red. c axial, $E_{a}$ (blue), bending, $E_{b}($ red $)$, and shear, $E_{s}$ (green), strain energy versus $\lambda$ (color figure online) 


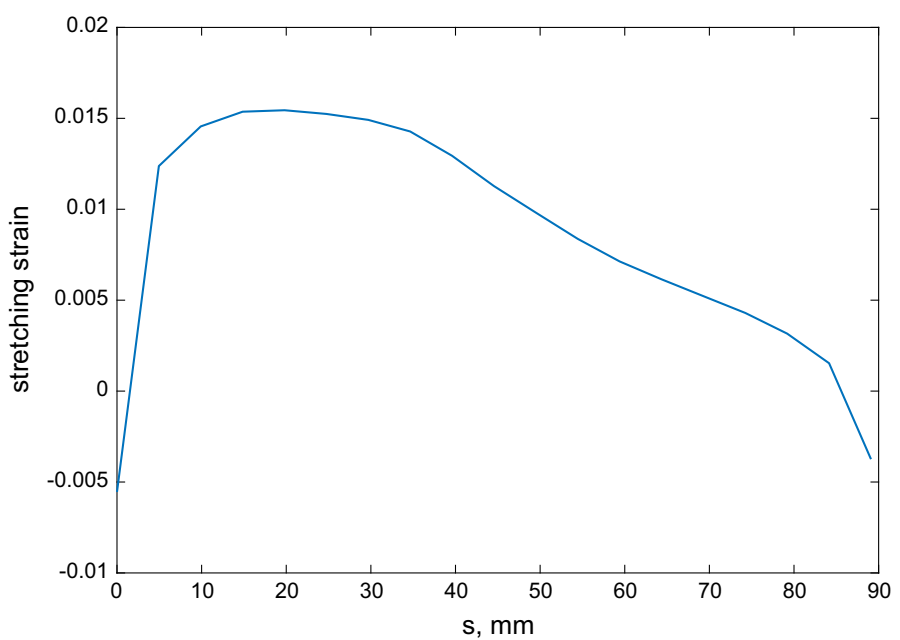

FIG. 10. Bias test performed by using a second gradient model: spring elongation along $x_{2}=\sqrt{2} \varepsilon+x_{1}$

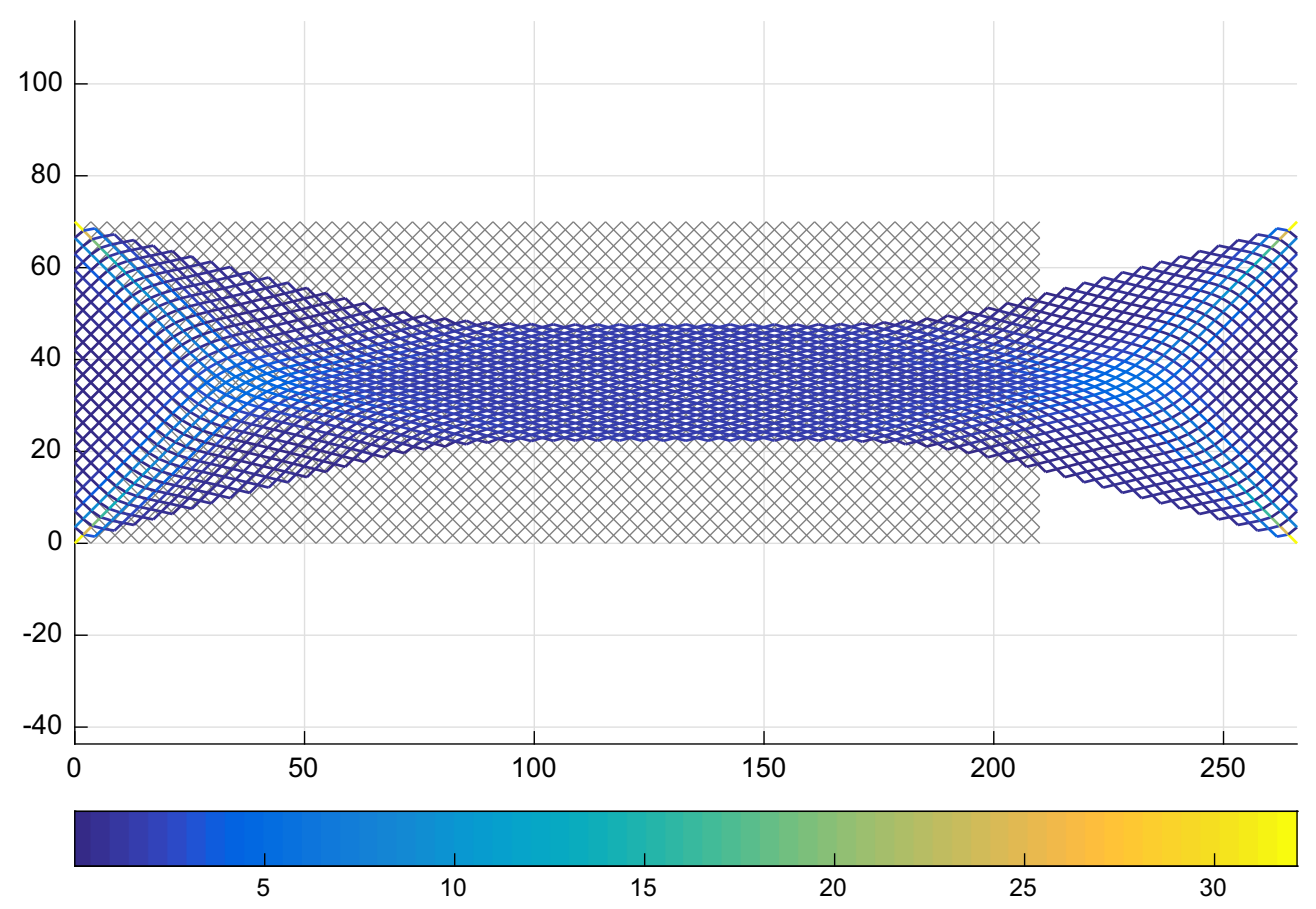

FIG. 11. Bias test for $n_{f}=20$ : reference configuration (gray), deformation and color bar of the internal forces on extensional springs (color figure online)

for the case $n_{f}=10$, a plot which shows the elongations of the extensional springs which lie on the line $x_{2}=\sqrt{2} \varepsilon+x_{1}$.

The same test was performed by using a second gradient model, see Figs. 8, 9 and 10 which show the good agreement between the discrete and the continuum models. This is more evident when the numerical 


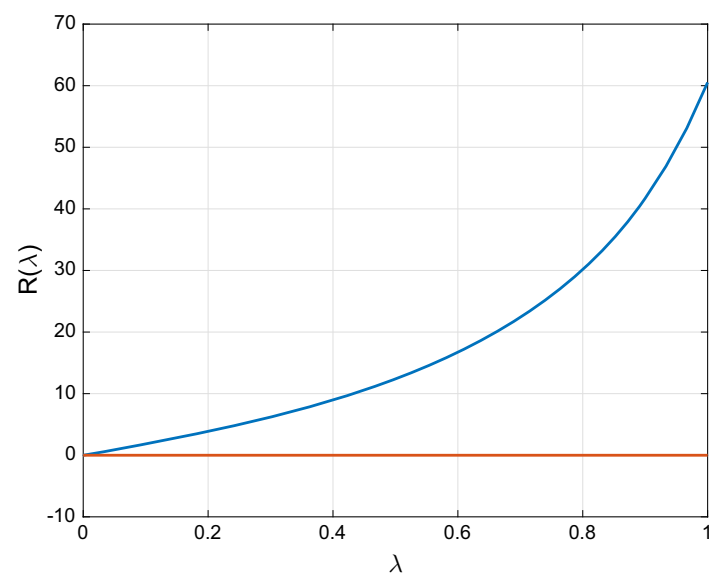

(a)

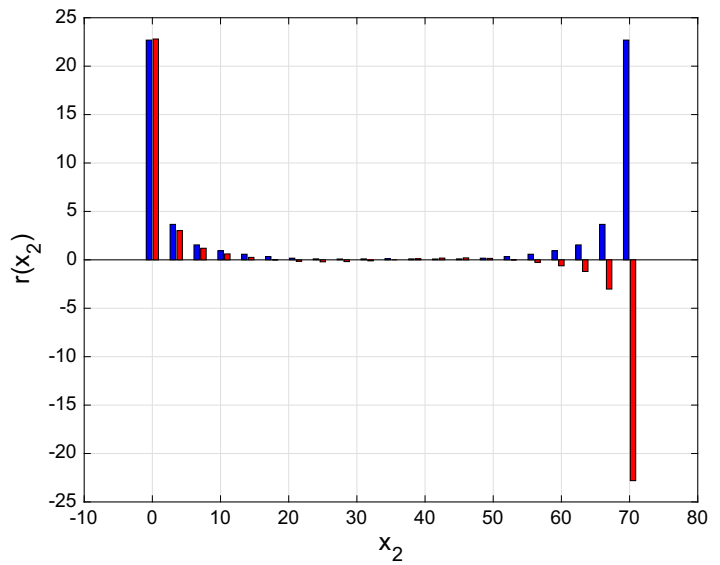

(b)

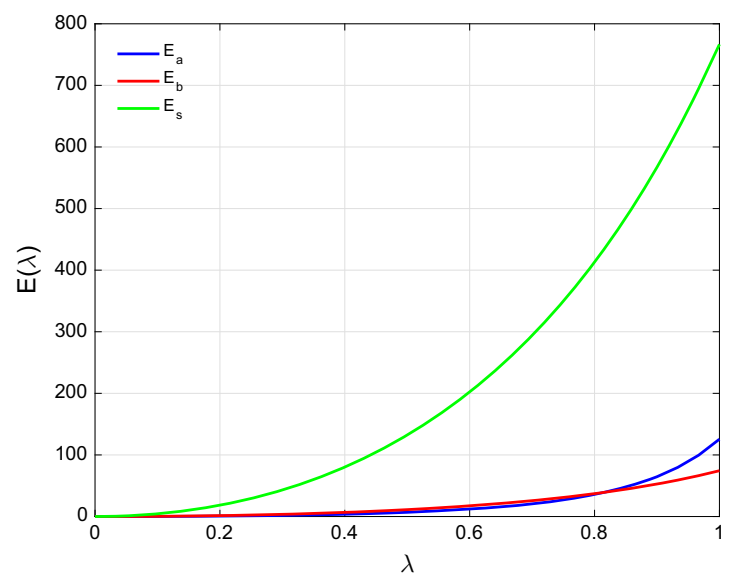

(c)

FIG. 12. Bias test for $n_{f}=20$ : global reaction of constraints $R(\lambda)$ (a), its density $r\left(x_{2}\right)$ when $\lambda=1$ (b) and strain energies (c). a $x_{1}$ component in blue and $x_{2}$ component in red. $\mathbf{b} x_{1}$ component in blue and $x_{2}$ component in red. c axial, E $E_{a}$ (blue), bending, $E_{b}($ red $)$, and shear, $E_{s}$ (green), strain energy versus $\lambda=\bar{u} / \bar{u}_{\max }$ (color figure online) 


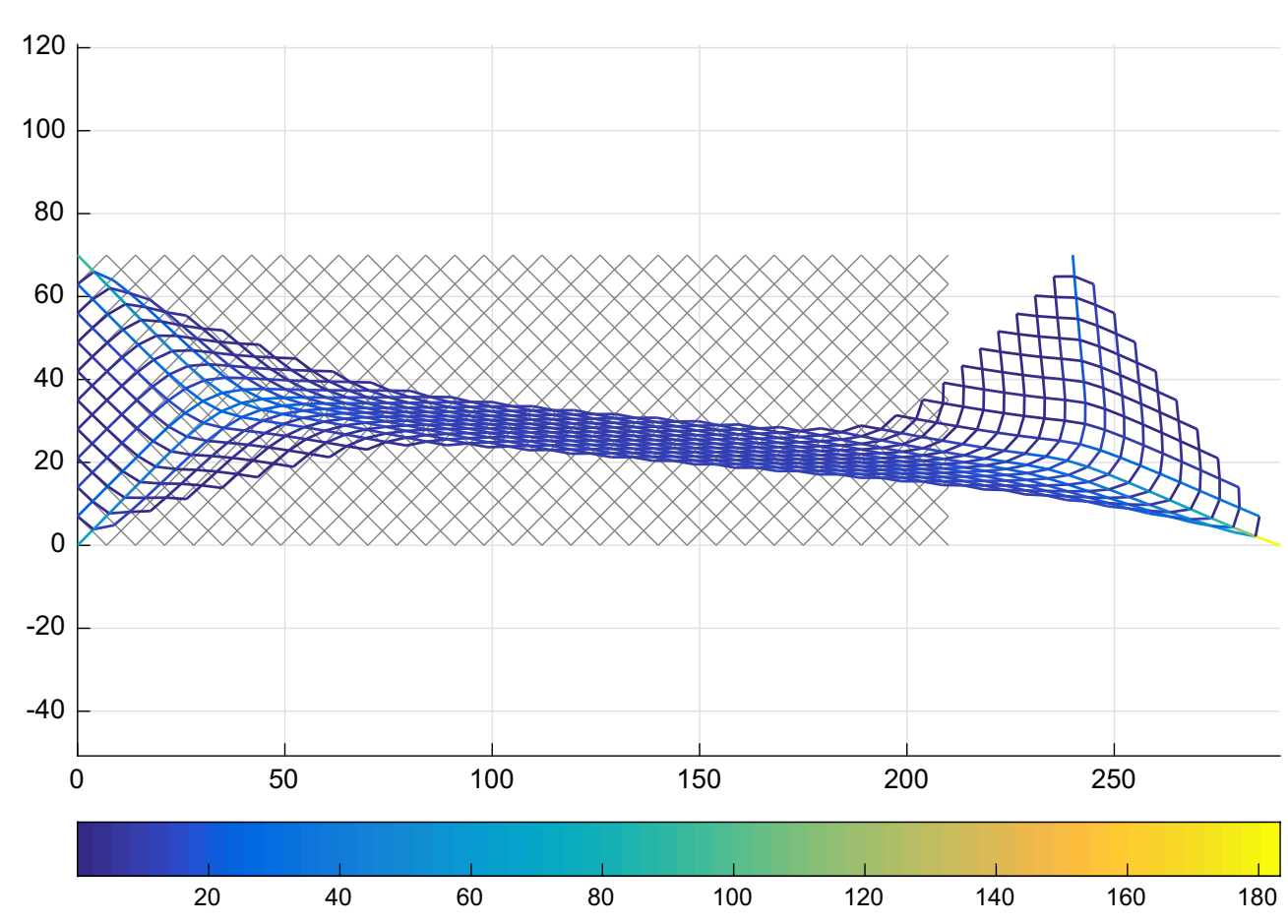

FIG. 13. Bending-extension test for $n_{f}=10$ : reference configuration (gray), deformation and color bar of the internal forces on extensional springs (color figure online)

results of the Hencky-type model for a pantographic structure with $n_{f}=20$ are considered, see Figs. 11 and 12 .

\subsection{Bending-extension test}

In this case, the imposed displacements are:

$$
\begin{aligned}
u_{1}\left(0, x_{2}\right) & =u_{2}\left(0, x_{2}\right)=u_{2}\left(3 \ell, x_{2}\right)=0, \\
\left.u_{1}\left(3 \ell, x_{2}\right)\right) & =80-\frac{5}{7} x_{2} \mathrm{~mm},
\end{aligned}
$$

which produce, for the case $n_{f}=10$, the deformation plot reported in Fig. 13 along with the color bar of the internal forces on extensional springs. Figure 14, as for the previous numerical test, reports the global reaction $R(\lambda)$, its density $r\left(x_{2}\right)$ and the strain energies subdivided in the extensional, bending and shear parts.

Figures 15 and 16 show, for the same test, the results obtained from a second gradient model confirming the reliability of the discrete model results.

\subsection{Shear-extension test}

Shear-extension test is defined by the following displacements:

$$
u_{1}\left(0, x_{2}\right)=u_{2}\left(0, x_{2}\right)=0,
$$




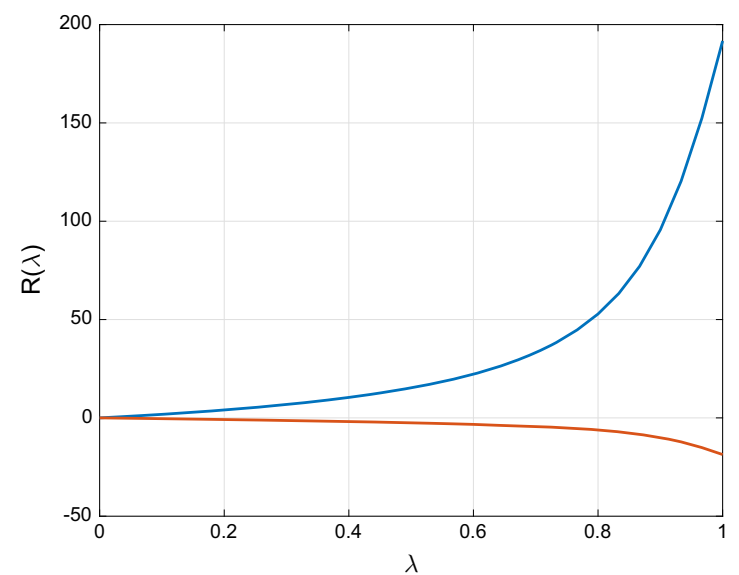

(a)

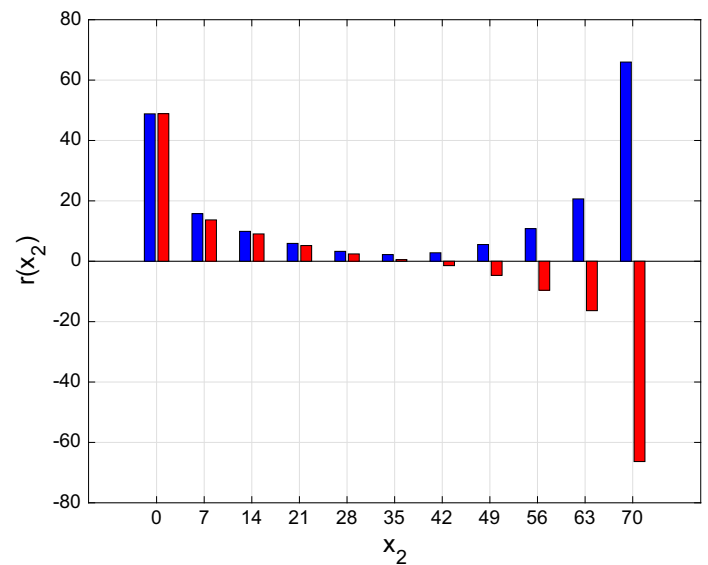

(b)

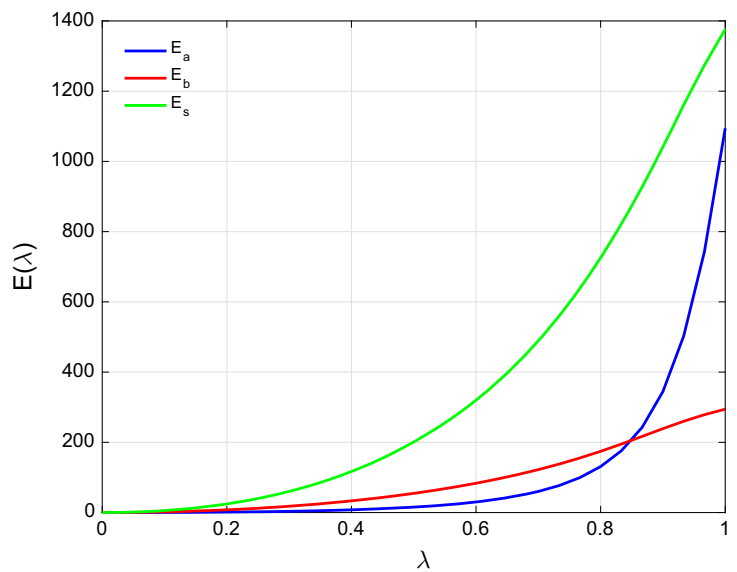

(c)

FIG. 14. Bending-extension test for $n_{f}=10$ : global reaction of constraints $R(\lambda)$ versus $\lambda=\bar{u} / \bar{u}_{\max }$ (a) and its density $r\left(x_{2}\right)$ when $\lambda=1$ (b) and strain energies (c). a $x_{1}$ component in blue and $x_{2}$ component in red. b $x_{1}$ component in blue and $x_{2}$ component in red. c axial, $E_{a}$ (blue), bending, $E_{b}$ (red), and shear, $E_{s}$ (green), strain energy versus $\lambda=\bar{u} / \bar{u}_{\text {max }}($ color figure online) 


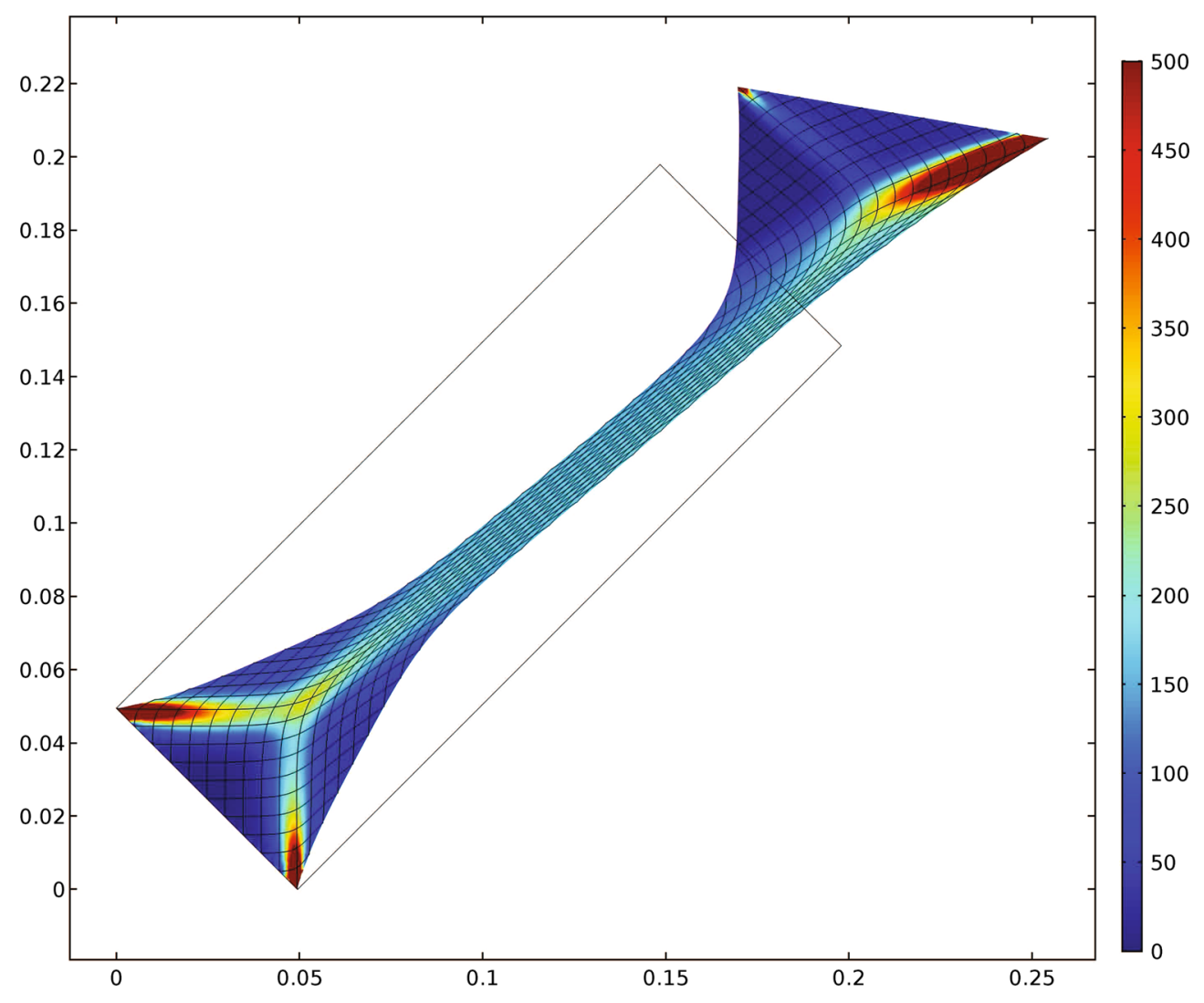

FIG. 15. Bending-extension test by using a second gradient model: reference configuration (gray), deformation and color bar of the strain energy (color figure online)

$$
\begin{aligned}
\left.u_{1}\left(3 \ell, x_{2}\right)\right) & =0.4 \ell=28 \mathrm{~mm}, \\
u_{2}\left(3 \ell, x_{2}\right) & =\ell=70 \mathrm{~mm},
\end{aligned}
$$

which produce, for the case $n_{f}=10$, the deformation plot reported in Fig. 17 along with the color bar of the internal forces on extensional springs. Analogously, Fig. 18 reports the global reaction $R(\lambda)$, its density $r\left(x_{2}\right)$ and the strain energies subdivided in the extensional, bending and shear parts.

\subsection{Extraction test}

The last numerical test is characterized by the following imposed displacements:

$$
\begin{aligned}
u_{1}(0,0) & =u_{2}(0,0)=u_{1}(0, \ell)=u_{2}(0, \ell)=0.0 \\
u_{1}(3 \ell, 0) & =u_{2}(3 \ell, 0)=0.8 \ell=56 \mathrm{~mm}, \\
u_{1}(3 \ell, \ell) & =u_{2}(3 \ell, \ell)=0.8 \ell=56 \mathrm{~mm},
\end{aligned}
$$

which produce, for the case $n_{f}=10$, the deformation plot reported in Fig. 19 along with the color bar of the internal forces on extensional springs. Finally, Fig. 20, reports the global reaction $R(\lambda)$, its density $r\left(x_{2}\right)$ and the strain energies subdivided in the extensional, bending and shear parts. 


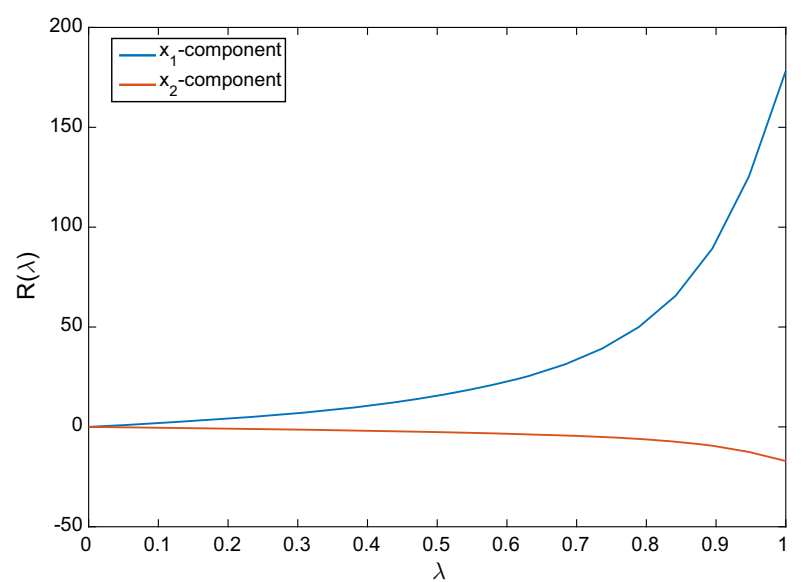

(a)

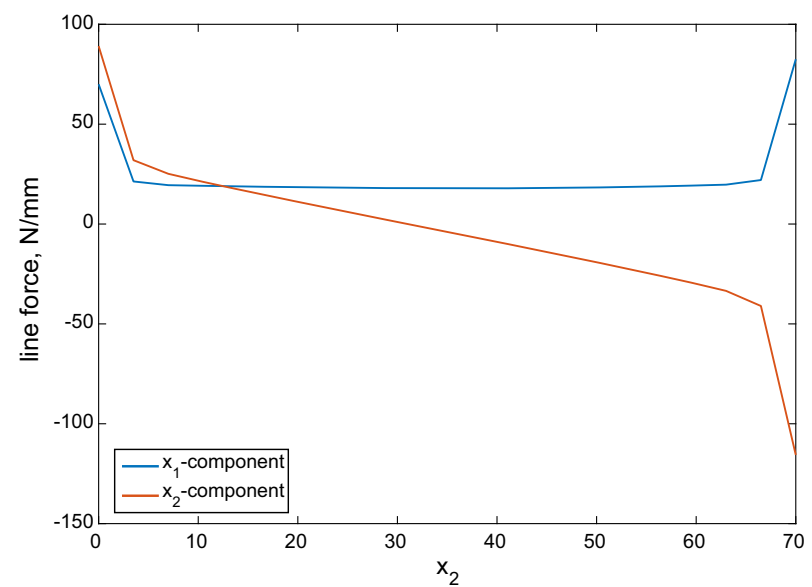

(b)

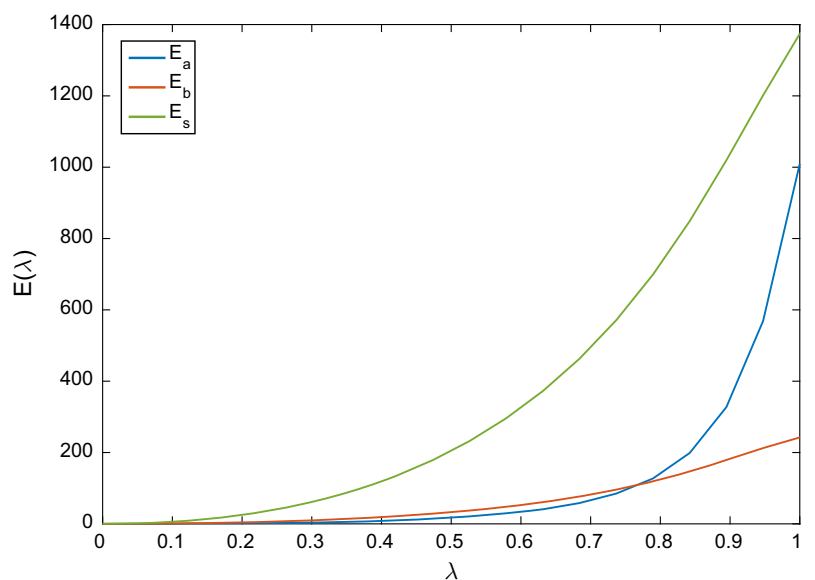

(c)

FIG. 16. Bending-extension test by using a second gradient model: global reaction of constraints $R(\lambda)(\mathbf{a})$, its density $r\left(x_{2}\right)$ when $\lambda=1$ (b) and strain energies (c). a $x_{1}$ component in blue and $x_{2}$ component in red. b $x_{1}$ component in blue and $x_{2}$ component in red. c axial, $E_{a}$ (blue), bending, $E_{b}\left(\right.$ red), and shear, $E_{s}$ (green), strain energy versus $\lambda=\bar{u} / \bar{u}_{\text {max }}($ color figure online) 


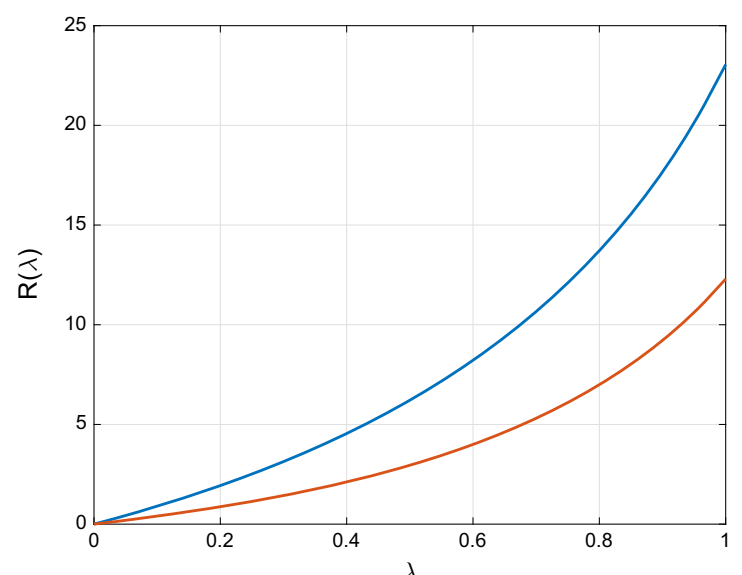

(a)

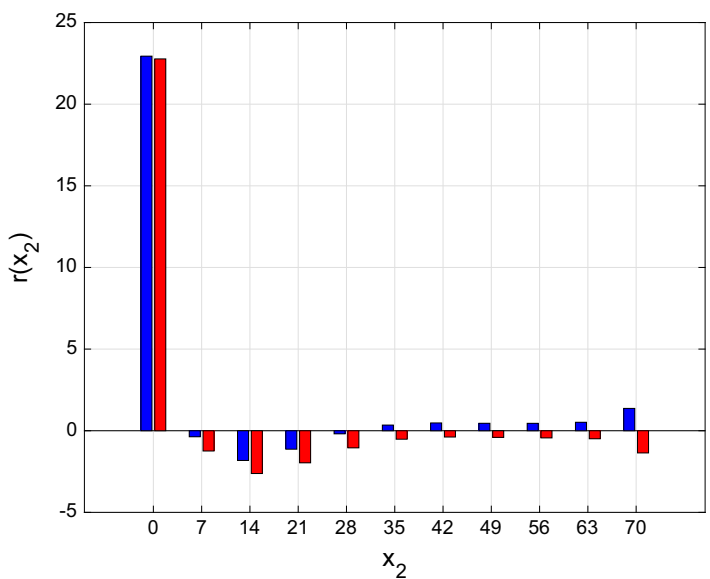

(b)

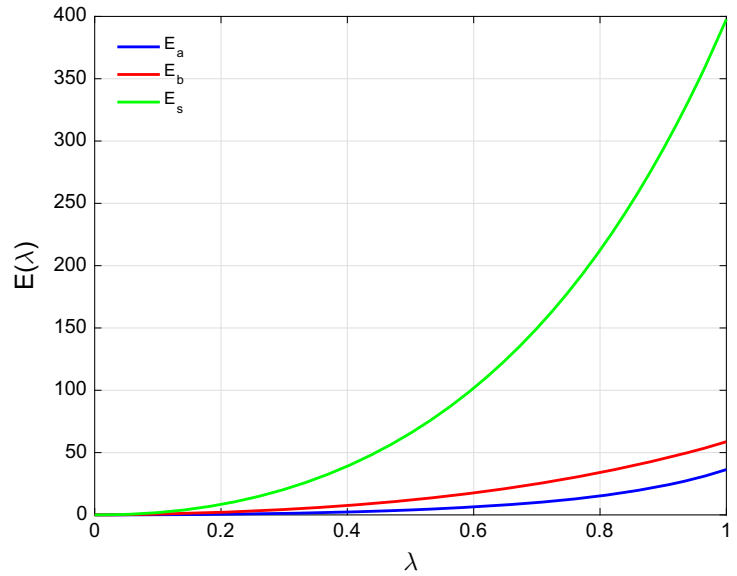

(c)

FIG. 18. Shear-extension test: global reaction of constraints $R(\lambda)$ versus $\lambda=\bar{u} / \bar{u}_{\max }(\mathbf{a})$, its density $r\left(x_{2}\right)$ when $\lambda=1$ (b) and strain energies for $n_{f}=10$ (c). a $x_{1}$ component in blue and $x_{2}$ component in red. b $x_{1}$ component in blue and $x_{2}$ component in red. c axial, $E_{a}$ (blue), bending, $E_{b}($ red $)$, and shear, $E_{s}$ (green), strain energy versus $\lambda=\bar{u} / \bar{u}_{\text {max }}($ color figure online) 


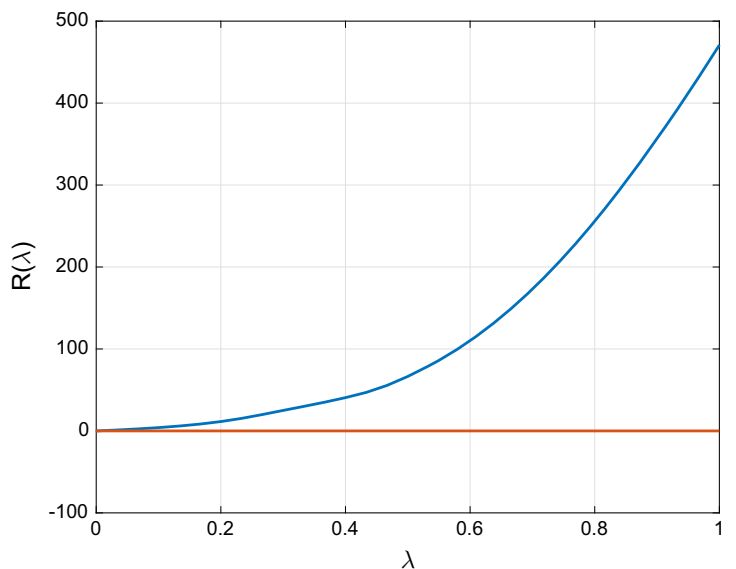

(a)

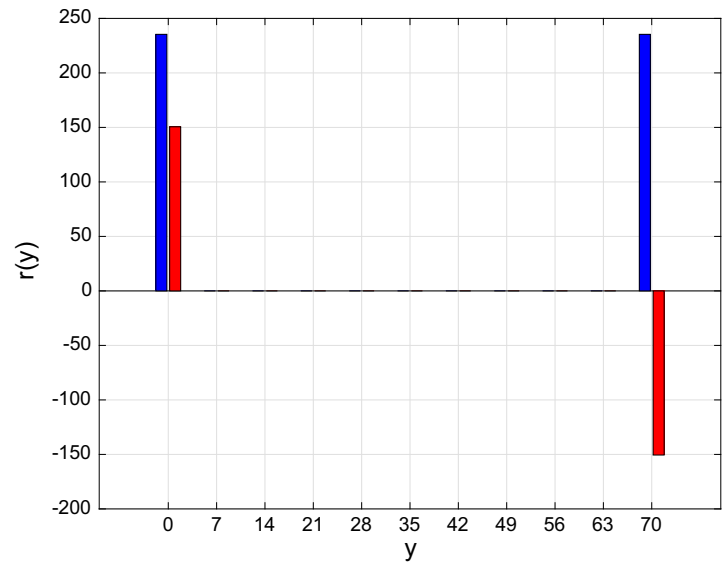

(b)

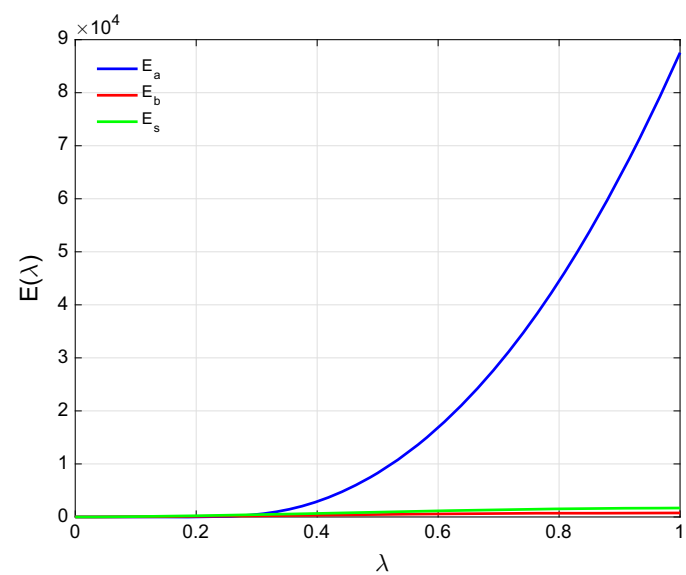

(c)

FIG. 20. Extraction test for $n_{f}=10$ : global reaction of constraints $R(\lambda)$ versus $\lambda=\bar{u} / \bar{u}_{\text {max }}(\mathbf{a})$, its density $r\left(x_{2}\right)$ when $\lambda=1$ (b) and strain energies (c). a $x_{1}$ component in blue and $x_{2}$ component in red, $\mathbf{b} x_{1}$ component in blue and $x_{2}$ component in red, c axial, $E_{a}$ (blue), bending, $E_{b}$ (red), and shear, $E_{s}$ (green), strain energy versus $\lambda=\bar{u} / \bar{u}_{\text {max }}$ (color figure online) 


\section{References}

1. Hencky, H.: Über die angenäherte Lösung von Stabilitätsproblemen im Raum mittels der elastischen Gelenkkette. Ph.D. thesis, Engelmann (1921)

2. dell'Isola, F., Giorgio, I., Pawlikowski, M., Rizzi, N.L.: Large deformations of planar extensible beams and pantographic lattices: Heuristic homogenisation, experimental and numerical examples of equilibrium. Proc. R. Soc. Lond. A Math. Phys. Eng. Sci. 472(2185) (2016)

3. dell'Isola, F., Steigmann, D., Della Corte, A.: Synthesis of fibrous complex structures: designing microstructure to deliver targeted macroscale response. Appl. Mech. Rev. 67(6), 060804 (2015)

4. dell'Isola, F., Della Corte, A., Greco, L., Luongo, A.: Plane bias extension test for a continuum with two inextensible families of fibers: a variational treatment with Lagrange multipliers and a perturbation solution. Int. J. Solids Struct. 81, 1-12 (2016)

5. dell'Isola, F., Lekszycki, T., Pawlikowski, M., Grygoruk, R., Greco, L.: Designing a light fabric metamaterial being highly macroscopically tough under directional extension: first experimental evidence. Z. Angew. Math. Phys. 66(6), 34733498 (2015)

6. Challamel, N., Kocsis, A., Wang, C.M.: Discrete and non-local elastica. Int. J. Non-Linear Mech. 77, 128-140 (2015)

7. Wang, C.M., Zhang, H., Gao, R.P., Duan, W.H., Challamel, N.: Hencky bar-chain model for buckling and vibration of beams with elastic end restraints. Int. J. Struct. Stab. Dyn. 15(7), 1540007 (2014)

8. Challamel, N., Zhang, Z., Wang, C.M.: Nonlocal equivalent continua for buckling and vibration analyses of microstructured beams. J. Nanomech. Micromech. 5, A4014004-1-A4014004-16 (2014)

9. Madeo, A., Della Corte, A., Greco, L., Neff, P.: Wave propagation in pantographic 2D lattices with internal discontinuities. Proc. Estonian Acad. Sci. 64(3S), 325-330 (2015)

10. Greco, L., Giorgio, I., Battista, A.: In plane shear and bending for first gradient inextensible pantographic sheets: numerical study of deformed shapes and global constraint reactions. Math. Mech. Solids (2016). doi:10.1177/1081286516651324

11. Placidi, L., Andreaus, U., Giorgio, I.: Identification of two-dimensional pantographic structure via a linear D4 orthotropic second gradient elastic model. J. Eng. Math. (2016). doi:10.1007/s10665-016-9856-8

12. Boisse, P., Hamila, N., Guzman-Maldonado, E., Madeo, A., Hivet, G., dell'Isola, F.: The bias-extension test for the analysis of in-plane shear properties of textile composite reinforcements and prepregs: a review. Int. J. Mater. Form. (2016). doi:10.1007/s12289-016-1294-7

13. Barbagallo, G., Madeo, A., Azehaf, I., Giorgio, I., Morestin, F., Boisse, P.: Bias extension test on an unbalanced woven composite reinforcement: experiments and modeling via a second-gradient continuum approach. J. Compos. Mater. (2016). doi:10.1177/0021998316643577

14. Cao, J., Akkerman, R., Boisse, P. et al.: Characterization of mechanical behavior of woven fabrics: experimental methods and benchmark results. Compos. Part A Appl. Sci. Manuf. 39(6), 1037-1053 (2008)

15. Harrison, P., Clifford, M.J., Long, A.C.: Shear characterisation of viscous woven textile composites: a comparison between picture frame and bias extension experiments. Compos. Sci. Technol. 64(10), 1453-1465 (2004)

16. Harrison, P., Abdiwi, F., Guo, Z., Potluri, P., Yu, W.R.: Characterising the shear-tension coupling and wrinkling behaviour of woven engineering fabrics. Compos. Part A Appl. Sci. Manuf. 43(6), 903-914 (2012)

17. Harrison, P.: Modelling the forming mechanics of engineering fabrics using a mutually constrained pantographic beam and membrane mesh. Compos. Part A Appl. Sci. Manuf. 81, 145-157 (2016)

18. D'Agostino, M.V., Giorgio, I., Greco, L., Madeo, A., Boisse, P.: Continuum and discrete models for structures including (quasi-) inextensible elasticae with a view to the design and modeling of composite reinforcements. Int. J. Solids Struct. 59, 1-17 (2015)

19. Caggegi, C., Pensée, V., Fagone, M., Cuomo, M., Chevalier, L.: Experimental global analysis of the efficiency of carbon fiber anchors applied over cfrp strengthened bricks. Constr. Build. Mater. 53, 203-212 (2014)

20. Grillo, A., Wittum, G., Tomic, A., Federico, S.: Remodelling in statistically oriented fibre-reinforced materials and biological tissues. Math. Mech. Solids 20(9), 1107-1129 (2015)

21. Federico, S., Gasser, T.C.: Nonlinear elasticity of biological tissues with statistical fibre orientation. J. R. Soc. Interface $7(47), 955-966(2010)$

22. Grillo, A., Federico, S., Wittum, G.: Growth, mass transfer, and remodeling in fiber-reinforced, multi-constituent materials. Int. J. Non-Linear Mech. 47(2), 388-401 (2012)

23. Federico, S., Grillo, A., La Rosa, G., Giaquinta, G., Herzog, W.: A transversely isotropic, transversely homogeneous microstructural-statistical model of articular cartilage. J. Biomech. 38(10), 2008-2018 (2005)

24. Federico, S., Grillo, A.: Elasticity and permeability of porous fibre-reinforced materials under large deformations. Mech. Mater. 44, 58-71 (2012)

25. Engheta, N., Ziolkowski, R.W.: Metamaterials: Physics and Engineering Explorations. Wiley, New York (2006)

26. Zouhdi, S., Sihvola, A., Vinogradov, A.P.: Metamaterials and Plasmonics: Fundamentals, Modelling, Applications. Springer, Berlin (2008) 
27. Del Vescovo, D., Giorgio, I.: Dynamic problems for metamaterials: review of existing models and ideas for further research. Int. J. Eng. Sci. 80, 153-172 (2014)

28. Misra, A., Poorsolhjouy, P.: Granular micromechanics based micromorphic model predicts frequency band gaps. Contin. Mech. Thermodyn. 28(1), 215-234 (2016)

29. Auriault, J.-L., Boutin, C.: Long wavelength inner-resonance cut-off frequencies in elastic composite materials. Int. J. Solids Struct. 49(23), 3269-3281 (2012)

30. Boutin, C.: Acoustics of porous media with inner resonators. J. Acoust. Soc. Am. 134(6), 4717-4729 (2013)

31. Placidi, L., Giorgio, I., Della Corte, A., Scerrato, D.: Euromech 563 Cisterna di Latina 17-21 March 2014 Generalized continua and their applications to the design of composites and metamaterials: a review of presentations and discussions. Math. Mech. Solids (2015). doi:10.1177/1081286515576948

32. Placidi, L., Rosi, G., Giorgio, I., Madeo, A.: Reflection and transmission of plane waves at surfaces carrying material properties and embedded in second-gradient materials. Math. Mech. Solids 19(5), 555-578 (2014)

33. Rosi, G., Giorgio, I., Eremeyev, V.A.: Propagation of linear compression waves through plane interfacial layers and mass adsorption in second gradient fluids. Z. Angew. Math. Mech. 93(12), 914-927 (2013)

34. Berezovski, A., Giorgio, I., Della Corte, A.: Interfaces in micromorphic materials: wave transmission and reflection with numerical simulations. Math. Mech. Solids 21(1), 37-51 (2016)

35. Abd-alla, A.N., Giorgio, I., Galantucci, L., Hamdan, A.M., Del Vescovo, D.: Wave reflection at a free interface in an anisotropic pyroelectric medium with nonclassical thermoelasticity. Contin. Mech. Thermodyn. 28(1-2), 67-84 (2016)

36. Carcaterra, A., dell'Isola, F., Esposito, R., Pulvirenti, M.: Macroscopic description of microscopically strongly inhomogenous systems: a mathematical basis for the synthesis of higher gradients metamaterials. Arch. Ration. Mech. Anal. 218(3), 1239-1262 (2015)

37. Madeo, A., Della Corte, A., Giorgio, I., Scerrato, D.: Modeling and designing micro-and nano-structured metamaterials: towards the application of exotic behaviors. Math. Mech. Solids (2015). doi:10.1177/1081286515616036

38. Enakoutsa, K., Della Corte, A., Giorgio, I.: A model for elastic flexoelectric materials including strain gradient effects. Math. Mech. Solids 21(2), 242-254 (2016)

39. Giorgio, I., Galantucci, L., Della Corte, A., Del Vescovo, D.: Piezo-electromechanical smart materials with distributed arrays of piezoelectric transducers: current and upcoming applications. Int. J. Appl. Electromagn. Mech. 47(4), 1051$1084(2015)$

40. Cuomo, M., dell'Isola, F., Greco, L.: Simplified analysis of a generalized bias test for fabrics with two families of inextensible fibres. Z. Angew. Math. Phys. 67(3), 1-23 (2016)

41. Steigmann, D.J., Pipkin, A.C.: Equilibrium of elastic nets. Philos. Trans. R. Soc. Lond. A Math. Phys. Eng. Sci. 335(1639), 419-454 (1991)

42. Pipkin, A.C.: Some developments in the theory of inextensible networks. Q. Appl. Math. 38(3), 343-355 (1980)

43. Pipkin, A.C.: Plane traction problems for inextensible networks. Q. J. Mech. Appl. Math. 34(4), 415-429 (1981)

44. dell'Isola, F., Della Corte, A., Esposito, R., Russo, L.: Some cases of unrecognized transmission of scientific knowledge: from antiquity to Gabrio Piola's peridynamics and generalized continuum theories. In: Altenbach, H., Forest, S. (eds) Generalized Continua as Models for Classical and Advanced Materials. Springer, Cham, pp. 77-128 (2016)

45. dell'Isola, F., Andreaus, U., Placidi, L.: At the origins and in the vanguard of peridynamics, non-local and higher-gradient continuum mechanics: an underestimated and still topical contribution of Gabrio Piola. Math. Mech. Solids 20(8), 887$928(2015)$

46. Pideri, C., Seppecher, P.: A second gradient material resulting from the homogenization of an heterogeneous linear elastic medium. Contin. Mech. Thermodyn. 9(5), 241-257 (1997)

47. Camar-Eddine, M., Seppecher, P.: Determination of the closure of the set of elasticity functionals. Arch. Ration. Mech. Anal. 170(3), 211-245 (2003)

48. Dos Reis, F., Ganghoffer, J.F.: Construction of micropolar continua from the asymptotic homogenization of beam lattices. Comput. Struct. 112, 354-363 (2012)

49. Goda, I., Assidi, M., Ganghoffer, J.-F.: Equivalent mechanical properties of textile monolayers from discrete asymptotic homogenization. J. Mech. Phys. Solids 61(12), 2537-2565 (2013)

50. Cecchi, A., Rizzi, N.: Heterogeneous elastic solids: a mixed homogenization-rigidification technique. Int. J. Solids Struct. 38(1), 29-36 (2001)

51. Misra, A., Poorsolhjouy, P.: Granular micromechanics model of anisotropic elasticity derived from Gibbs potential. Acta Mech. 227(5), 1393-1413 (2016)

52. Misra, A., Poorsolhjouy, P.: Identification of higher-order elastic constants for grain assemblies based upon granular micromechanics. Math. Mech. Complex Syst. 3(3), 285-308 (2015)

53. Misra, A., Parthasarathy, R., Singh, V., Spencer, P.: Micro-poromechanics model of fluid-saturated chemically active fibrous media. Z. Angew. Math. Mech. 95(2), 215-234 (2015)

54. dell'Isola F., Placidi L.: Variational Principles are a Powerful Tool also for Formulating Field Theories, Volume 535 of Variational Models and Methods in Solid and Fluid Mechanics CISM Courses and Lectures. Springer, Berlin (2012) 
55. Federico, S., Grillo, A., Imatani, S., Giaquinta, G., Herzog, W.: An energetic approach to the analysis of anisotropic hyperelastic materials. Int. J. Eng. Sci. 46, 164-181 (2008)

56. Nadler, B., Steigmann D., J.: A model for frictional slip in woven fabrics. Compt. Rendus Mec. 331(12), 797-804 (2003)

57. Scerrato, D., Giorgio, I., Madeo, A., Liman, A., Darve, F.: A simple non-linear model for internal friction in modified concrete. Int. J. Eng. Sci. 80, 136-152 (2014)

58. Scerrato, D., Giorgio, I., Della Corte, A., Madeo, A., Limam, A.: A micro-structural model for dissipation phenomena in the concrete. Int. J. Numer. Anal. Methods Geomech. 39(18), 2037-2052 (2015)

59. Germain, P.: The method of virtual power in continuum mechanics. Part 2: microstructure. SIAM J. Appl. Math. 25(3), 556-575 (1973)

60. Mindlin, R.D.: Second gradient of strain and surface-tension in linear elasticity. Int. J. Solids Struct. 1(4), 417-438 (1965)

61. dell'Isola, F., Seppecher, P., Madeo, A.: How contact interactions may depend on the shape of cauchy cuts in n-th gradient continua: approach á la D'Alembert. Z. Angew. Math. Phys. 63(6), 1119-1141 (2012)

62. Carcaterra, A., dell'Isola, F., Esposito, R., Pulvirenti, M.: Macroscopic description of microscopically strongly inhomogenous systems: a mathematical basis for the synthesis of higher gradients metamaterials. Arch. Ration. Mech. Anal. (2015). doi: 10.1007/s00205-015-0879-5

63. Altenbach, J., Altenbach, H., Eremeyev, V.A.: On generalized Cosserat-type theories of plates and shells: a short review and bibliography. Arch. Appl. Mech. 80(1), 73-92 (2010)

64. Altenbach, H., Eremeyev, V.A.: On the linear theory of micropolar plates. Z. Angew. Math. Mech. 89(4), 242-256 (2009)

65. Gabriele, S., Rizzi, N., Varano V.: A 1D higher gradient model derived from Koiter's shell theory. Math. Mech. Solids 6 (2014). doi:10.1177/1081286514536721

66. dell'Isola, F., Maier, G., Perego, U., Andreaus, U., Esposito, R., Forest, S.: The complete works of Gabrio Piola: volume I-Commented English translation. Adv. Struct. Mater. (2014). doi:10.1007/978-3-319-00263-7

67. Navier, L.: Mémoire sur les lois de l'équilibre et du mouvement des corps solides élastiques. Mem. Inst. Nat. 3, 375-393 (1827)

68. Cercignani, C: Ludwig Boltzmann: The Man who Trusted Atoms. OUP Oxford, Oxford (2006)

69. Truesdell, C., Noll, W.: The Non-Linear Field Theories of Mechanics. Springer, Berlin (2004)

70. Kuhn, T.S.: The Structure of Scientific Revolutions. University of Chicago Press, Chicago (2012)

71. Russo, L.: Forgotten Revolution: How Science was Born in 300 BC and Why it Had to be Reborn. Springer, Berlin (2013)

72. de Solla Price D.: Gears from the Greek. The Antikythera mechanism: a calendar computer from ca. 80 BC. Trans. Am. Philos. Soc. 64(7), 1-70 (1974)

73. Bilotta, A., Formica, G., Turco, E.: Performance of a high-continuity finite element in three-dimensional elasticity. Int. J. Numer. Methods Biomed. Eng. (Commun. Numer. Methods Eng.) 26, 1155-1175 (2010)

74. Cazzani, A., Stochino, F., Turco, E.: An analytical assessment of finite elements and isogeometric analysis of the whole spectrum of Timoshenko beams. Z. Angew. Math. Mech. 1-25 (2016). doi:10.1002/zamm.201500280

75. Cazzani, A., Malagù, M., Turco, E.: Isogeometric analysis: a powerful numerical tool for the elastic analysis of historical masonry arches. Contin. Mech. Thermodyn. 28(1), 139-156 (2016)

76. Cazzani, A., Malagù, M., Turco, E., Stochino, F.: Constitutive models for strongly curved beams in the frame of isogeometric analysis. Math. Mech. Solids 21(2), 182-209 (2016). doi:10.1177/1081286515577043

77. Greco, L., Cuomo, M.: An implicit $G^{1}$ multi patch B-spline interpolation for Kirchhoff-Love space rod. Comput. Methods Appl. Mech. Eng. 269, 173-197 (2014)

78. Cuomo, M., Contraffatto, L., Greco, L.: A variational model based on isogeometric interpolation for the analysis of cracked bodies. Int. J. Eng. Sci. 80, 173-188 (2014)

79. Greco, L., Cuomo, M.: Consistent tangent operator for an exact Kirchhoff rod model. Contin. Mech. Thermodyn. 27(4), 861-877 (2015)

80. Greco, L., Cuomo, M.: An isogeometric implicit G1 mixed finite element for Kirchhoff space rods. Comput. Methods Appl. Mech. Eng. 298, 325-349 (2016)

81. Alibert, J.-J., Della Corte, A.: Second-gradient continua as homogenized limit of pantographic microstructured plates: a rigorous proof. Z. Angew. Math. Phys. 66(5), 2855-2870 (2015)

82. Andreaus, U., Casini, P.: Friction oscillator excited by moving base and colliding with a rigid or deformable obstacle. Int. J. Non-Linear Mech. 37(1), 117-133 (2002)

83. Andreaus, U., Baragatti, P., Placidi, L.: Experimental and numerical investigations of the responses of a cantilever beam possibly contacting a deformable and dissipative obstacle under harmonic excitation. Int. J. Non-Linear Mech. 80, 96106 (2016)

84. Cuomo, M., Ventura, G.: Complementary energy approach to contact problems based on consistent augmented Lagrangian formulation. Math. Comput. Model. 28(4), 185-204 (1998)

85. D'Annibale, F., Rosi, G., Luongo, A.: On the failure of the 'similar piezoelectric control' in preventing loss of stability by nonconservative positional forces. Z. Angew. Math. Phys. 66(4), 1949-1968 (2015)

86. D'Annibale, F., Rosi, G., Luongo, A.: Linear stability of piezoelectric-controlled discrete mechanical systems under nonconservative positional forces. Meccanica 50(3), 825-839 (2015) 
87. Piccardo, G., Pagnini, L.C., Tubino, F.: Some research perspectives in galloping phenomena: critical conditions and post-critical behavior. Contin. Mech. Thermodyn. 27(1-2), 261-285 (2015)

88. Luongo, A., Paolone, A., Piccardo, G.: Postcritical behavior of cables undergoing two simultaneous galloping modes. Meccanica 33(3), 229-242 (1998)

89. Rizzi, N., Varano, V., Gabriele, S.: Initial postbuckling behavior of thin-walled frames under mode interaction. ThinWalled Struct. 68, 124-134 (2013)

90. AminPour, H., Rizzi, N.: A one-dimensional continuum with microstructure for single-wall carbon nanotubes bifurcation analysis. Math. Mech. Solids 4, 168-181 (2015)

91. Eremeyev, V.A., Pietraszkiewicz, W.: Material symmetry group and constitutive equations of micropolar anisotropic elastic solids. Math. Mech. Solids (2015). doi:10.1177/1081286515582862

92. Eremeyev, V.A., Pietraszkiewicz, W.: Material symmetry group of the non-linear polar-elastic continuum. Int. J. Solids Struct. 49(14), 1993-2005 (2012)

Emilio Turco

DADU, Università degli Studi di Sassari

Sassari, Italy

e-mail: emilio.turco@uniss.it

Francesco dell'Isola

DISG, Università di Roma "La Sapienza"

Rome, Italy

e-mail: francesco.dellisola@uniroma1.it

Antonio Cazzani

DICAAR, Università degli Studi di Cagliari

Cagliari, Italy

e-mail: antonio.cazzani@unica.it

Nicola Luigi Rizzi

Dipartimento di Architettura, Università di Roma Tre

Rome, Italy

e-mail: nicolaluigi.rizzi@uniroma3.it 